\title{
LA RIFORMA DEI REATI SESSUALI IN GERMANIA DEL 2016
}

\author{
A REFORMA DOS DELITOS SEXUAIS NA ALEMANHA DO ANO 2016
}

\section{THE GERMAN REFORM OF SEXUAL OFFENCES OF 2016}

FRANCESCO MACRì

http://orcid.org/0000-0001-7658-6173 / https://www.penalecontemporaneo.it/autori/799-francesco-macri / francesco.macri@unifi.it

Assegnista di ricerca in diritto penale presso il Dipartimento di Scienze Giuridiche dell'Università degli Studi di Firenze e Professore a contratto di diritto penale del corso di Scienze della Sicurezza presso la Scuola Marescialli e Brigadieri Carabinieri di Firenze. Profesor honorario en el nivel de Excelencia Academica presso la Facoltà di Diritto della Università San Carlos de Guatemala.

Firenze, Itália.

\begin{abstract}
RIASSUNTO
Il legislatore tedesco ha nel 2016 riformato incisivamente la legislazione penale sessuale. La norma di maggiore impatto giuridico e simbolico è il § $177 / 1$ StGB, che sancisce - per la prima volta in un grande ordinamento di Civil Law - la punibilità degli atti sessuali “meramente dissensuali”. Ulteriore modifica rilevante è l'incriminazione degli atti sessuali commessi "a sorpresa", così come quella delle molestie sessuali. È stato poi introdotto un peculiare delitto "accessorio" ( $\$ 184 \mathrm{j}$ StGB) che sanziona la mera partecipazione ad un gruppo che induca uno dei membri a commettere un reato sessuale. Siffatta previsione, peraltro, si connota per una strumentalizzazione simbolica del diritto penale che non appare accompagnata da un potenziamento funzionale della tutela della libertà sessuale. In un'ottica complessiva, tuttavia, va osservato come la suddetta ombra (così come altre minori) sia affiancata da importanti luci, a partire dalla svolta "consensualistica" del Sexualstrafrecht tedesco, che ad avviso dello scrivente consentono un giudizio globalmente positivo - pur con talune riserve - sulla riforma.
\end{abstract}

Parole chiave: codice penale tedesco; dissenso sessuale; molestie sessuali; reati sessuali; stupro.

\section{RESUMO}

O legislador alemão reformou profundamente o direito penal sexual em 2016. A mudança mais importante, legal e midiaticamente, é o § 177/1 StGB, que - pela primeira vez em um grande sistema jurídico de Civil Law - estabelece o princípio segundo o qual o dissenso da vítima é suficiente para a criminalização de um ato sexual. Outra inovação relevante é a penalização dos atos sexuais impostos "com surpresa" para a vítima, assim como a introdução do novo delito de “importunação sexual”. Além disso, a legislação penal reformada inclui também um novo "delito acessório" (o do 184j) destinado a criminalizar o mero fato de ser membro de um grupo quando este induza um ou mais dos outros membros a cometer um delito sexual: essa norma, entretanto, parece realizar uma instrumentalização simbólica do direito penal sem aumentar efetivamente a proteção da liberdade sexual, mas implicando uma potencial violação dos princípios básicos de garantia do direito penal. Globalmente, porém, a avaliação da reforma do Sexualstrafrecht é positiva, sobretudo em relaçào à melhor proteção da autodeterminação sexual das pessoas.

Palavras-chave: direito penal alemão, dissenso sexual, importunação sexual, crimes sexuais, estupro.

\section{ABSTRACT}

In 2016, the German penal legislator has sharply reformed the law of sexual offences. The most important provision, legally but also from a media/symbolic point of view, is the $\S 177 / 1 \mathrm{StGB}$, that states - for the first time in a major Civil Law country - the principle that the contrary will (disagreement) of the victim is sufficient for the criminalization of a sexual conduct. Another relevant innovation is the criminalization of sexual acts imposed "with surprise" to the victim, as well as the introduction of a new offence of "sexual harassment". Moreover, the new legislation comprises a peculiar new "side offence" ( $(184 \mathrm{j}$ ) aimed at punishing the mere fact of being a member of a group that "induces" 
one (or more) of the other members to commit a sexual offence: this provision, however, appears to be a "symbolic" instrumentalization of criminal law, with no effective increase in protection of sexual freedom, while at the same time carrying a potential violation of fundamental guarantees in criminal law. In the whole, however, the reform of the Sexualstrafrecht is to be positively evaluated, especially in regards to victims' rights of sexual self determination.

Keywords: german criminal law; sexual disagreement, sexual harassment, sexual offences, rape.

\section{SUMÁRIO}

INTRODUZIONE (INTRODUÇÃO); 1 QUADRO GENERALE DELLA LEGGE DI RIFORMA; 1.1 La genesi della riforma: $i$ fatti di Colonia e Amburgo (1.1.2016); 1.2 L'iter legislativo; 1.3 I testi della vecchia e della nuova disciplina a confronto ; 2 IL SEXUALSTRAFRECHT TEDESCO PREVIGENTE; 2.1 L'evoluzione storica del diritto penale sessuale tedesco ante reforma; 2.2 La nozione cardine di "atto sessuale"("Sexuelle Handlung") nel diritto penale sessuale tedesco anteriore al 10.11.2016; 2.3 I mezzi di costrizione "tradizionali" del Sexualstrafrecht previgente: violenza e minaccia grave; $2.4 \mathrm{La}$ nuova modalità costrittiva, c.d. Ausnutzungsvariante, introdotta nel 1997; 3 LA DISCIPLINA VIGENTE DEL SEXUALSTRAFRECHT QUALE RISULTANTE DALLA RIFORMA DEL 2016; 3.1 La centralità del dissenso nella fattispecie "base" di aggressione sessuale del \$ 177/1 StGB; 3.2 Le nuove figure criminose di abuso sessuale, ed equiparate, ex $\$ 177 / 2$ StGB; 3.2.1 Gli abusi delle condizioni di inferiorità fisica o psichica (\$ 177/2, nn. 1 e 2 StGB); 3.2 .2 Le aggressioni sessuali mediante sorpresa (\$ 177/2, n. 3 StGB). - 3.2.3 Le aggressioni sessuali “minacciose" (\$ 177/2, nn. 4 e 5); 3.3 Lo "Stupro" e gli altri delitti sessuali di maggiore gravità di cui ai \$§ 177/4 - 177/8 StGB; 3.4 Le fattispecie (autonome) attenuate del \$ 177/9; 3.5 II nuovo delitto di "Molestie sessuali" ex \$ 184i StGB; 3.6. La controversa incriminazione ( $\$ 184 \mathrm{j} \mathrm{StGB}$ ) della "partecipazione indiretta" ai reati sessuali; 4 CONSIDERAZIONI CONCLUSIVE SULLA RIFORMA DEL SEXUALSTRAFRECHT TEDESCO NEL SUO COMPLESSO (CONCLUSÃO); 4.1 Aspetti positivi; 4.2 Aspetti negativi; BIBLIOGRAFIA (REFERÊNCIAS)

\section{INTRODUZIONE}

Il legislatore tedesco ha recentemente proceduto ad una incisiva riforma della legislazione penale sessuale: il 10 novembre 2016 è difatti entrata in vigore una disciplina su molti punti innovativa. La norma di maggiore impatto, giuridico ma anche mediatico/simbolico, è senz'altro il § $177 / 1$ StGB, che sancisce - per la prima volta in un grande ordinamento di Civil Law - la punibilità degli atti sessuali “meramente dissensuali”, cioè commessi “contro la volontà riconoscibile" della vittima, senza necessità di violenza, minaccia ecc. Ulteriore modifica rilevante è poi l'incriminazione di nuovo conio degli atti sessuali commessi “a sorpresa”, così come quella delle molestie sessuali. Entrambe sono dovute all'intento del legislatore fortemente pressato dall'opinione pubblica - di impedire che si ripetano fatti come quelli del Capodanno 2016 a Colonia e ad Amburgo, notte nella quale si verificarono aggressioni e molestie sessuali di gruppo ai danni di centinaia di donne, e in relazione alle quali non vi sono state condanne sostanziali a causa - oltre che di difficoltà nell'identificazione dei responsabili - del 
mancato utilizzo di violenza o minaccia (intesi secondo la giurisprudenza del BGH) da parte degli autori, i quali invece si erano avvalsi delle condizioni di affollamento e confusione.

In risposta a tali eventi, è stato altresi introdotto un peculiare delitto "accessorio" ( $\S$ 184j StGB)che sanziona sostanzialmente la mera partecipazione ad un gruppo, qualora quest'ultimo induca uno dei membri a commettere un reato sessuale: non essendo richiesto alcun contributo di compartecipazione (morale o materiale, ai sensi dei \$§ 25-27 StGB), la fattispecie in questione appare - a meno di interventi giurisprudenziali "ortopedici" - in patente contrasto con i principi generali, sancendo una sorta di "responsabilità per fatto altrui". Siffatta previsione, pertanto, si connota per una strumentalizzazione simbolica del diritto penale che, a differenza di altre norme, non appare accompagnata da un adeguato potenziamento funzionale della tutela del bene giuridico di riferimento (la libertà sessuale).

In un'ottica complessiva, tuttavia, va osservato come la suddetta ombra (così come altre minori) sia affiancata da importanti luci, a partire dalla svolta "consensualistica" del Sexualstrafrecht tedesco, che ad avviso dello scrivente consentono un giudizio globalmente positivo - pur con talune riserve - sulla riforma.

\section{QUADRO GENERALE E GENESI DELLA LEGGE DI RIFORMA}

Il 10 novembre 2016 è entrata in vigore la nuova disciplina dei "Delitti contro la libertà sessuale” (\$§ 174 ss.) di cui al codice penale tedesco (Strafgesetzbuch - StGB), frutto dalle modifiche apportate dalla 50. legge di modifica del codice penale, intitolata "Potenziamento della tutela della libertà sessuale"1.

Con l'intervento normativo in commento, il legislatore tedesco ha implementato il principio - oggetto di ampie attenzioni mediatiche - comunemente etichettato come "No significa no" ("Nein heißt Nein"), in virtù del quale è sufficiente superare il dissenso della vittima all'approccio sessuale ai fini della rilevanza penale dello stesso, in contrapposizione alla previgente legislazione, che richiedeva - in parallelismo con le normative penali sessuali dei maggiori ordinamenti di Civil Law, a partire da quello italiano - determinate modalità di costrizione (violenza, minaccia grave ecc.).

\footnotetext{
1 Fünfzigstes Gesetz zur Änderung des Strafgesetzbuches - "Verbesserung des Schutzes der sexuellen Selbstbestimmung", il cui testo è reperibile sul sito internet ufficiale del Bundesgesetzblatt (BGBl corrispondente alla nostra Gazzetta Ufficiale), aprendo a p. 2460 il file pdf consultabile all'indirizzo http://www.bgbl.de/xaver/bgbl/start.xav?startbk=Bundesanzeiger_BGBl\#_bgbl_\%2F\%2F*\%5B\%40attr_id\% 3D\%27bgbl116052.pdf\%27\%5D_1478686819712>.
} 
Ulteriore profilo caratterizzante l'appena menzionata riforma normativa è stata l'introduzione di una nuova fattispecie criminosa di "Molestie sessuali" (\$184i StGB, "Sexuelle Belästigung”), affiancata da un'apposita e controversa disposizione (\$184j) mirata a estendere la punibilità di coloro che partecipino a gruppi "che inducano taluno alla commissione di un reato sessuale" (ex §§ 177 o 184i StGB) ben oltre quanto previsto dalle ordinarie norme sul concorso di persone di cui ai §§ 25 - 31 StGB.

Si consideri peraltro come - dai primi commenti, per lo più rilasciati a testate giornalistiche - a fronte di un generale plauso nella società civile, svariati giuristi tedeschi abbiano incisivamente criticato taluni profili della riforma, e principalmente l'insufficiente determinatezza di svariati concetti (es. "volontà riconoscibile"), la innecessarietà di molte previsioni, e la 'strumentalizzazione simbolica' del diritto penale ${ }^{2}$. Tali critiche verranno approfondite nei successivi paragrafi.

Tralasciando per il momento le molestie sessuali, da un punto di vista tecnico, la svolta 'consensualistica' del diritto penale sessuale tedesco si è tradotta nei seguenti interventi modificativi (approfonditi nel prosieguo):

1) II § 177 StGB, prima intitolato "Costrizione sessuale; Stupro" ("Sexuelle Nötigung; Vergewaltigung") è stato reintitolato "Aggressione sessuale; Costrizione sessuale; Stupro" ("Sexueller Übergriff; sexuelle Nötigung; Vergewaltigung”), ampliandosi così notevolmente fino ad includere ogni tipo di aggressione o abuso sessuale.

2) II $\S 177 / 1$ (= $\S 177$, Absatz [comma] $\left.1^{\circ}\right)$ StGB è stato rivoluzionato, incentrando il delitto base di "Costrizione sessuale" sul compimento di atti sessuali "contro la riconoscibile volontà" ("gegen den erkennbaren Willen”) della vittima: si è pertanto passati da un modello 'vincolato' di disciplina dei reati sessuali (che richiedeva violenza, minaccia ecc.) ad un modello 'consensuale' (rectius 'consensuale limitato' o 'dissensuale', per non confondere il dissenso di cui

\footnotetext{
${ }^{2}$ In primis v. FISCHER, Thomas. Zum letzen Mal: Neinheißt Nein. Zeit online, 28 giugno 2016. Disponibile in <http://www.zeit.de/gesellschaft/zeitgeschehen/2016-06/rechtspolitik-sexualstrafrecht-nein-heisstnein-fischer-im-recht.> V. altresi, in senso tendenzialmente critico FROMMEL, Monika. Das neue Gesetz ist "Unsinn". Deutschlandradio Kultur: 7 luglio 2016. Disponibile in <http:/ / www. deutschlandradiokultur. de/sexualstrafrecht-das-neue-gesetz-istunsinn.1008.de.html?dram:article_id=359391>; HOVEN, Elisa; FISCHER, Thomas. Wir erleben eine Moralisierung des Rechts. LTO - Legal Tribune Online, 31 ottobre 2016. Disponibile in <http://www.lto.de/recht/hintergruende/h/sexualstrafrecht-reform-elisa-hoven-thmoas-fischer/>; MÜLLER, Henning Ernst. Der "erkennbare" Wille nach dem neuen Sexualstrafrechts - erkennbar fehlerhaft. Pubblicato l'8 luglio 2016 e disponibile in <www.community.beck.de $>$. A favore della riforma invece v. HÖRNLE,Tatjana. Kopfschütteln oder Weinen genügt. Taz.de: 5 luglio 2016. Disponibile in http://www.taz.de/!5315782/>.
} 
al § $177 / 1$ StGB con la mancanza di consenso sui cui si impernia la disciplina dei reati sessuali nei paesi di Common Law $^{3}$ ).

3) Il delitto di "Abuso sessuale di persona incapace di resistere" ${ }^{4}$, contemplato dal vecchio § $179 \mathrm{StGB}$, è stato espunto dal testo codicistico. Le ipotesi criminose ivi previste (commissione di atti sessuali su persone in condizioni di inferiorità fisica o psichica), difatti, sono state ricollocate nell'attuale $§ 177 \mathrm{StGB}$, ed affiancate (nell'Abs. 2) da due nuove figure criminose di abuso sessuale, meno gravemente sanzionate.

4) Sono stati tipizzati ( $\$ 177 / 2$ StGB) nuovi reati di aggressione sessuale mediante minaccia (contemplando altresì una seconda tipologia di 'costrizione ambientale') e, soprattutto, si è introdotta nella medesima disposizione l'incriminazione degli atti sessuali 'repentini' (cioè perpetrati mediante sorpresa) $)^{5}$.

5) Il § 177 StGB è stato ampliato (forse in modo eccessivo) da 5 a 9 Absätze (commi), di modo da racchiudere in sé tutte le ipotesi di aggressione/abuso sessuale, ordinate da quelle meno gravi (Abss. 1 e 2), punite con pena da 6 mesi a 5 anni di reclusione; a quelle più gravi (Abs. 8), sanzionate con la reclusione da 5 a 15 anni). Nel dettaglio, le figure criminose tipizzate - ed i relativi compassi sanzionatori edittali - possono essere schematizzate nel modo seguente:

A) $\S 177 / 1$ e 177/2 - da 6 mesi a 5 anni di reclusione:

- Atti sessuali 'dissensuali' (“contro la riconoscibile volontà del soggetto”).

- Atti sessuali su soggetti incapaci di consentire per condizioni transeunti).

- Atti sessuali su soggetti incapaci di consentire a causa delle proprie condizioni fisiche o psichiche.

- Atti sessuali perpetrati avvalendosi di un momento di sorpresa.

- Atti sessuali realizzati in una situazione nella quale la vittima tema di subire un male rilevante (“empfindliches Übel”) in caso di resistenza.

- Atti sessuali commessi mediante minaccia di un male rilevante.

B) $\S \S 177 / 4$ e 177/5 - da 1 anno a 15 anni di reclusione:

- Atti sessuali su soggetti incapaci di consentire a causa di una malattia ("Krankheit") o di una condizione di disabilità ("Behinderung”).

\footnotetext{
${ }^{3}$ A partire dalla normativa penale sessuale inglese (e gallese), di cui al Sexual Offences Act 2003, di cui a titolo esemplificativo - la section 3 ("Aggressione sessuale" ["Sexual Assault"] recita: "(1) Una persona (A) commette un reato se: (a) tocca intenzionalmente un' altra persona (B), (b) il toccamento è sessuale, (c) B non acconsente al toccamento, (d) A non ritiene ragionevolmente che B acconsenta".

4 "Sexueller Mißbrauch widerstandsunfähiger Personen".

${ }^{5}$ Che si verifica, secondo il riformato testo normativo (\$177/2, n. 3, StGB), ove l'autore commetta l'atto sessuale "sfruttando un momento di sorpresa" (“ein Überraschungsmoment ausnutzt”).
} 
- Atti sessuali commessi mediante violenza.

- Atti sessuali commessi con minaccia di un pericolo attuale ("gegenwärtige Gefahr") per la vita (“Leben”) o l'integrità fisica (“Leib”) della persona offesa.

- Atti sessuali perpetrati mediante "approfittamento di una situazione in cui la vittima è indifesa alla mercé dell'agente" (previgente $§ 177 / 1$ n. 3 StGB $^{6}$ ).

C) § $177 / 6$ - da 2 anni a 15 anni di reclusione.

Casi di particolare gravità, tra i quali il legislatore ha espressamente menzionato - con la tecnica del 'esempio di norma' ("Regelbeispiel”) ${ }^{7}$ - i seguenti :

- Atti sessuali penetrativi, cioè ipotesi di "Stupro" ("Vergewaltigung”).

- Atti sessuali commessi “insieme” (“gemeinschaflich”) da più persone.

D) $§ 177 / 7$ - da 3 anni a 15 anni di reclusione:

- Atti sessuali perpetrati portando con sé un'arma, o comunque uno strumento pericoloso, ove l'agente si avvalga degli stessi per vincere o impedire la resistenza del soggetto passivo, oppure qualora l'aggressore ponga - con tali strumenti - la vittima in grave pericolo di subire rilevanti danni alla salute.

E) $§ 177 / 8$ - da 5 anni a 15 anni di reclusione:

- Atti sessuali perpetrati facendo uso di un'arma, o comunque di uno strumento pericoloso, ove la vittima subisca gravi maltrattamenti fisici durante la commissione del fatto, oppure si venga a trovare in pericolo di vita.

\subsection{La genesi della riforma: i fatti di Colonia e Amburgo (1.1.2016)}

Le motivazioni principali che hanno spinto il Parlamento tedesco ad approvare - in tempi molto celeri, come si vedrà a breve - una così rilevante 'novellazione' della normativa

${ }^{6}$ Trattasi della riproposizione della cosìddetta "Ausnützungsvariante", introdotta nel 1997 con la 33. Strafänderungsgesetz. Al riguardo sia consentito il rinvio, per approfondimenti, a MACRì, Francesco. Costrizione 'ambientale' agli atti sessuali: la tutela del dissenso tra legalità ed esigenze repressive in un raffronto tra codice penale italiano e StGB tedesco. Rivista Italiana Diritto e Procedura Penale, $\mathrm{n}^{\circ} 4$, 2007. p. 1492.

${ }^{7}$ Sulle problematiche della Regelbeispieltechnik, mediante la quale si orienta il giudice nell'applicazione di cornici edittali differenziate per casi concreti caratterizzati da un diverso disvalore di condotta o di evento rispetto alle fattispecie base, e la loro funzione di "criteri di commisurazione della pena" ("Strafzumessungsregeln", cosi' RENZIKOWSKI, Joachim. Das Sexualstrafrecht nach dem 6. Strafrechtsreformgesetz. NStZ, 1999. P. 378. V. nella letteratura italiana PAPA, Michele; PALAZZO, Francesco. Lezioni di diritto penale comparato. 3a Ed. Torino: Giappichelli, 2013. p. 80.; in quella tedesca, ex plurimis, EISELE, Jörg. Die Regelbeispielsmethode im Strafrecht. Tuebingen: Mohr Siebeck, 2004; FASSIN, Bernhard. Strazumessung im Urteilstenor: Regelbeispiele besonders und minder schwerer Fälle. Shaker Verlag: Aachen, 1995. 
penale a tutela della libertà sessuale non vanno ricercate nelle richieste o appelli all'intervento legislativo da parte di accademici e pratici del diritto, bensì nelle vibranti proteste levatesi ad opera di ampi settori della popolazione, e in primis dai movimenti femministi, a seguito dell'ondata di violenze e molestie sessuali scatenatasi - su scala mai vista prima - durante la notte di Capodanno 2016 a Colonia e ad Amburgo (e in misura minore anche in altre città, tedesche e non).

In tale notte, difatti, secondo il rapporto ufficiale della Polizia federale tedesca e stando ai dati raccolti dalla commissione d'inchiesta "Silvester", oltre 1.000 donne furono vittime di aggressioni sessuali (650 a Colonia e 400 ad Amburgo) perpetrate da circa 2.000 aggressori (per lo più agenti in gruppo), quasi tutti cittadini extracomunitari, e in maggioranza provenienti dal nord-Africa: al luglio 2016 (data del rapporto ufficiale), però, solo 120 uomini risultavano indagati, e unicamente 4 condannati (tra l'altro a pene molto lievi) ${ }^{8}$.

Ciò che si verificò in tale notte fu la perpetrazione di attacchi sessuali sistematici da parte di gruppi di uomini a danno di donne, avvalendosi i primi dell'affollamento e della confusione tipici della notte di San Silvestro (particolarmente accentuati in città come Colonia e Amburgo) ${ }^{9}$. Nel rapporto menzionato viene evidenziata altresì la non adeguata gestione dell'emergenza da parte delle forze di polizia, non preparate a fronteggiare simili aggressioni, e che in molti casi neanche si resero conto della gravità ed estensione delle stesse ${ }^{10}$. Le principali criticità rilevate dagli investigatori, peraltro, sono state di natura 'probatoria', e dovute all'assenza di immagini chiare degli aggressori, e all'impossibilità da parte della maggioranza delle vittime di riconoscerli o descriverli nel dettaglio.

\footnotetext{
8 Trattasi del rapporto della Polizia federale anticipato, il 10 luglio 2016, dal quotidiano Süddeutsche Zeitung e dalle emittenti Ndr e Wdr, di cui ampi stralci possono trovarsi - in lingua italiana - nell'articolo “Colonia, violenze di Capodanno. Metà aggressori arrivati nel 2015", pubblicato dal Corriere della Sera in data 11 luglio 2016, e reperibile all'indirizzo web <http://www.corriere.it/esteri/16_luglio_12/coloniaviolenze-capodanno-meta-aggressori-arrivati-2015-88917860-47a6-11e6-af4e-15bff4e09cf7.shtml>.

${ }^{9}$ Stando alla relazione della succitata commissione d'inchiesta "Silvester", a Capodanno furono commessi in tutto 642 reati puramente sessuali, per i quali sono stati indagati 47 sospetti; e in 239 casi le molestie furono accompagnate da furti o borseggi, con 73 relativi indagati. Tali gravissimi episodi tra l'altro avvennero, oltre che a Colonia e Amburgo, anche a Stoccarda, Düsseldorf ed altre città.

${ }^{10}$ Dalle immagini diffuse, tra l'altro, si evince chiaramente la riluttanza della polizia ad intervenire con la dovuta fermezza nei confronti di soggetti (per lo più nordafricani o mediorientali), verosimilmente per il timore di subire accuse di abusi o addirittura di razzismo. Ciò è dovuto purtroppo anche all'opera di un ampio settore del mondo accademico, culturale e giornalistico europeo, che tende a criminalizzare a priori - a causa di errori e abusi da parte di singoli membri - l'operato delle forze di polizia nel loro complesso, e a tacciare indiscriminatamente come illegittima e abusiva l'azione coercitiva delle pubbliche autorità.
} 
Sotto il profilo della normativa penale, si rilevò da più parti come l'allora vigente legislazione impedisse un'adeguata risposta penale, posto che i §§ 177 e 179 StGB non contemplavano l'incriminazione delle aggressioni sessuali commesse mediante sorpresa, in assenza di violenza, minaccia, o delle altre condizioni che verranno a breve analizzate. Nella notte di violenza de quo, invece, gran parte delle aggressioni avvennero con tali modalità, come dimostrato dai numerosi video postati online di donne accerchiate e toccate sessualmente (in taluni casi veri in modo penetrativo) da gruppi di uomini ai quali non potevano sfuggire, vista la calca e l'affollamento: in particolare fece scalpore, nei media d'oltralpe, la notizia di taluni aggressori arrestati ad Amburgo in quanto ritratti da un fotografo amatoriale, ed immediatamente rilasciati proprio per la non sussumibilità delle intrusioni sessuali perpetrate sotto alcuna fattispecie penale.

A seguito dei fatti appena descritti, e della loro risonanza mediatica, subito iniziarono le proteste e le manifestazioni popolari, guidate e coordinate da esponenti dei movimenti femministi. Lo slogan utilizzato sin dall'inizio - e poi riproposto dai media a seguito dell'approvazione della riforma, racchiudendo in esso la 'sintesi icastica' del nuovo diritto penale sessuale tedesco - è stato all'uopo “No significa No" (“Nein heißt Nein”). L'obiettivo, in linea teorica del tutto condivisibile, dei movimenti tedeschi pro riforma dei primi mesi del 2016 era dunque quello di ottenere una nuova legislazione penale sessuale che garantisse una tutela piena alla libertà sessuale, attribuendo rilevanza unica al consenso in quanto tale del destinatario dell'approccio sessuale, pur in assenza di una - all'epoca normativamente prescritta - caratterizzazione violenta, minacciosa ecc. della condotta.

\subsection{L'iter legislativo}

Preso atto delle richieste e delle proteste provenienti da ampi settori della popolazione, è stato il Governo tedesco di grande coalizione, presieduto dalla cancelliera Angela Merkel, a prendere l'iniziativa in modo tempestivo sin da febbraio/marzo $2016^{11}$ : l'attivismo dell'Esecutivo si è pertanto tradotto nel fondamentale progetto di legge (Gesetzentwurf) 18/8210 del 25 aprile 2016.

\footnotetext{
11 Probabilmente anche per rimediare, in vista delle elezioni del 2017, all'emorragia di consensi - con conseguente avanzata del partito di destra AFD (Alternative für Deutschland) - scaturita dall'adozione nel 2015 (poi in gran parte revocata) di politiche migratorie di grande apertura molto contestate, soprattutto a seguito delle violenze di Colonia e Amburgo, gran parte delle quali furono perpetrate da nordafricani arrivati clandestinamente in Germania nei mesi immediatamente precedenti al Capodanno 2016.
} 
Tale draft prevedeva già la criminalizzazione delle aggressioni/abusi sessuali realizzati pur in assenza di violenza, minaccia ecc. - avvalendosi della repentinità / sorpresa (Überraschung) della condotta, ma nel complesso non comportava una 'rivoluzione' dell'assetto normativo vigente ${ }^{12}$. Il suddetto progetto, infatti, interveniva soprattutto sulla fattispecie criminosa di "Abuso sessuale di persona incapace di resistere" ("Sexueller Mißbrauch widerstandsunfähiger Personen") ex $\S 179$ StGB, ricollocando in essa l'ipotesi di atti sessuali realizzati mediante "approfittamento di una situazione in cui la vittima è indifesa alla mercé dell'agente" di cui al previgente $\S 177 / 1$ n. 3 StGB $^{13}$; e introducendo, sempre nel $\S 179$, due nuove figure criminose, date dagli "atti sessuali repentini / a sorpresa" e dagli "atti sessuali realizzati in una situazione nella quale la vittima tema di subire un male rilevante in caso di resistenza".

Il progetto di legge $18 / 8210$ ha costituito dunque il testo di partenza, ma è stato successivamente modificato a seguito dei lavori della commissione parlamentare competente e del plenum del Bundestag (Camera dello Stato Federale), le quali hanno provveduto soprattutto a recepire le osservazioni formulate dal Bundesrat (Camera delle Regioni [Länder]) con l'Atto contenente Raccomandazioni (Unterrichtung) del 1. giugno 2016 (Drucksache 18/862614). Il Bundesrat (che in materia ha un potere di interlocuzione, ma non di codecisione ${ }^{15}$ ) dopo aver affermato la totale condivisione della finalità governativa di estendere la tutela penale della libertà sessuale, ha all'uopo formulato tre principali osservazioni:

1) La mancata previsione (nel progetto governativo 18/8210) della rilevanza del semplice dissenso della vittima all'approccio sessuale, richiedendosi in esso quanto meno la repentinità dell'atto sessuale non condiviso, dà luogo al permanere di una "lacuna di punibilità" ("Strafbarkeitslücke") di cui sarebbe auspicabile la rimozione, essendo in contrasto con gli intenti dichiarati della riforma.

\footnotetext{
12 In questa sede non è possibile esaminare nel dettaglio tutte le peculiarità di tale progetto di legge (e neanche degli altri presentati nel corso dell'iter legislativo), ma si rimanda per approfondimenti alla consultazione del testo e della relazione di accompagnamento dello stesso, reperibile online (in formato pdf) all'indirizzo <http://dip21.bundestag.de/dip21/btd/18/082/1808210.pdf>.

13 Trattasi della riproposizione della cosiddetta "Ausnützungsvariante", introdotta nel 1997 con la 33. Strafänderungsgesetz. Al riguardo si rimanda a quanto già detto nel primo paragrafo (e agli scritti citati in nota), e a quanto si aggiungerà altresì nei successivi paragrafi di questa trattazione.

${ }^{14} \mathrm{Il}$ testo integrale contenente le raccomandazioni e osservazioni del Bundesrat è consultabile all'indirizzo web < http://dip21.bundestag.de/dip21/btd/18/086/1808626.pdf $>$.

${ }^{15}$ La Camera delle Regioni (Bundesrat) tedesca è un organo costituzionale attraverso la quale i Länder incidono sull'esercizio del potere legislativo ed esecutivo dello Stato federale tedesco, ed altresì sui rapporti di quest'ultimo con l'Unione Europea.
} 
2) Sarebbe preferibile inserire, per ragioni sistematiche ma non solo, nell'ambito sulla fattispecie di “Costrizione sessuale” (§ 177 StGB) e non di quella di “Abuso sessuale” (§ 179 StGB), le nuove figure criminose a tutela della libertà sessuale.

3) Sarebbe opportuna l'incriminazione, in un autonomo paragrafo del testo punitivo, delle molestie sessuali, posto che - in considerazione degli attuali orientamenti esegetici della Corte Suprema tedesca (Bundesgerichtshof) - le vigenti disposizioni non garantiscono la repressione penalistica di tutti i toccamenti sessuali meritevoli di sanzione criminale (citando espressamente il caso dei "molestatori” [“Grabscher”] di Colonia e Amburgo).

Queste (così come le altre) raccomandazioni della Camera dei Länder sono state pienamente recepite dal Bundestag, il quale ha proceduto ad approvare (con ampio margine) la riforma codicistica, in prima lettura, il 7 luglio 2016, e ha successivamente apportato ulteriori modifiche, che sono state oggetto di voto favorevole in una seconda deliberazione avvenuta il 9 settembre.

Da ultimo, il Bundesrat ha deliberato in dato 23 settembre 2016 - a larga maggioranza l'approvazione del testo da ultimo licenziato dall'altra Camera ("Entwurf eines Gesetzes zür Änderung ses Strafgesetzbuches - Verbesserung des Schutzes der sexuellen Selbst Bestimmung”: Bundesrat Drucksache 463/16 ${ }^{16}$ ): a seguito della firma del Presidente della Repubblica Joachim Gauch il 4 novembre, e della pubblicazione sul BGBl il 9 novembre $^{17}$, il nuovo diritto penale sessuale è entrato in vigore il 10 novembre $2016^{18}$.

\subsection{I testi della vecchia e della nuova normativa a confronto}

Tab.1: Dir. penale sessuale tedesco (aggressioni e abusi sessuali) vigente e ante reforma

\begin{tabular}{|l|l|}
\hline \multicolumn{1}{|c|}{ StGB: Legislazione vigente } & \multicolumn{1}{c|}{ StGB: Legislazione anteriore } \\
\hline $\begin{array}{l}\text { \$177 - AGGRESSIONE SESSUALE, COSTRIZIONE } \\
\text { SESSUALE, STUPRO }\end{array}$ & \$177 - COSTRIZIONE SESSUALE, STUPRO \\
$\begin{array}{l}\text { (1) Chiunque contro la volontà riconoscibile di } \\
\text { un'altra persona compie sulla stessa, o fa } \\
\text { compiere dalla stessa atti sessuali, oppure la } \\
\text { determina a compiere o subire atti sessuali }\end{array}$ & $\begin{array}{l}\text { 1) mediante violenza, } \\
\text { vita o l'integrità fisica o }\end{array}$ \\
\hline
\end{tabular}

\footnotetext{
${ }^{16}$ Il testo legislativo approvato in via definitiva dal Bundesrat (e in primis dal Bundestag il 2 settembre) è reperibile online al seguente indirizzo web: <http://dipbt.bundestag.de/dip21/brd/2016/0463-16.pdf>. $17<$ http: / / www.bgbl.de/xaver/bgbl/start.xav?startbk=Bundesanzeiger_BGBl\#_bgbl_\%2F\%2F*\%5B\%40attr_i d\%3D\%27bgbl116s2460.pdf\%27\%5D_1478687968391>.

18 Il codice penale tedesco (Strafgesetzbuch), nel testo attualmente vigente, è consultabile sul sito ufficiale (gestito dal Ministero federale della Giustizia) <http://www.gesetze-im-internet.de/stgb/>.
} 
con/da una terza persona, é punito con la reclusione da 6 mesi a 5 anni.

(2) La medesima pena si applica a chiunque compie su di un'altra persona, o fa compiere alla stessa, atti sessuali, oppure la determina a compiere o subire atti sessuali con/da una terza persona, qualora

1. l'autore approfitti di una situazione in cui che tale persona non sia in grado di formare o esprimere una volontà contraria,

2. l'autore abusi di una situazione in cui la capacità di formare o esprimere la volontà dell'altra persona, a causa della condizione fisica o psichica di quest'ultima, sia fortemente limitata (presupponendo che egli abbia ottenuto il consenso della persona agli atti sessuali),

3. l'autore sfrutti un momento di sorpresa,

4. l'autore si approfitti di una situazione nella quale la vittima tema di subire un male rilevante in caso di resistenza,

5. l'autore costringa la persona a compiere o subire atti sessuali mediante la minaccia di un male rilevante.

(3) Il tentativo è punibile.

(4) Si applica la pena della reclusione non inferiore ad 1 anno qualora l'incapacità di formare o esprimere la volontà derivi da una malattia o da un handicap della vittima.

(5)Si applica la reclusione non inferiore a 1 anno qualora l'autore

1. adoperi violenza nei confronti della vittima,

2. minacci la vittima di un pericolo attuale per la vita o l' integrità fisica o

3. si approfitti di una situazione in cui la vittima è indifesa alla mercé dell' agente.

(6) Nei casi di particolare gravità si applica la pena della reclusione non inferiore ad anni 2 . Si versa in casi di particolare gravità , di regola, qualora:

1. I'autore si congiunga carnalmente con la vittima, o compia o faccia compiere su di sé analoghi atti sessuali, tali da umiliarla in maniera particolarmente intensa e consistenti, segnatamente, in una penetrazione del corpo della vittima (stupro), oppure

2. più persone concorrano nella commissione del fatto.

(7) Si applica la pena della reclusione non inferiore ad anni 3 qualora il colpevole

1. porti con sé un 'arma od un altro oggetto pericoloso,

2. tale porto sia finalizzato ad impedire la
3. approfittandosi di una situazione in cui la vittima è indifesa alla mercé dell'agente,

costringe un' altra persona a subire atti sessuali dall'autore o da un terzo, o a compiere atti sessuali sull'autore o su un terzo, é punito con la reclusione non inferiore ad anni 1 .

(2) Nei casi di particolare gravità si applica la pena della reclusione non inferiore ad anni 2 . Si versa in casi di particolare gravità , di regola, qualora

1. l'autore si congiunga carnalmente con la vittima, o compia o faccia compiere su di sè analoghi atti sessuali, tali da umiliarla in maniera particolarmente intensa e consistenti, segnatamente, in una penetrazione del corpo della vittima (stupro), oppure

2. più persone concorrano nella commissione del fatto.

(3) Si applica la pena della reclusione non inferiore ad anni 3 qualora il colpevole

1. porti con sè un 'arma od un altro oggetto pericoloso,

2. tale porto sia finalizzato ad impedire la resistenza di un' altra persona mediante la violenza o la minaccia della stessa, o

3. ponga la vittima, mediante la commissione del fatto, in grave pericolo di subire rilevanti danni alla salute.

(4) Si applica la pena della reclusione non inferiore ad anni cinque qualora il colpevole

1. faccia uso, durante la commissione del fatto, di un 'arma o di altro oggetto pericoloso

2. la vittima, durante la commissione del fatto a) subisca gravi maltrattamenti o

b) si venga a trovare in pericolo di vita.

(5) Nei casi di minore gravità dell'Abs. 1 si applica la pena della reclusione da mesi 6 ad anni 5 , nei casi di minore gravitá degli Abs. 3 e 4 si applica la pena della reclusione da anni 1 ad anni 10.

\section{\$ 179 - ABUSO SESSUALE}

\section{PERSONA INCAPACE DI RESISTERE}

(1) Chiunque abusa di una persona incapace di resistere

1. a causa di una malattia o di un disturbo psichico, inclusi le sindromi da dipendenza e i gravi disturbi della coscienza

2. fisicamente

in modo tale da sfruttare tale incapacità per far compiere o subire all' incapace atti sessuale é punito con la pena della reclusione da mesi 6 ad anni 
resistenza di un'altra persona mediante la violenza o la minaccia della stessa, o

3. ponga la vittima, mediante la commissione del fatto, in grave pericolo di subire rilevanti danni alla salute.

Alla stessa pena soggiace chi abusa di una persona incapace di resistere (ai sensi dell' Abs. 1) in modo tale da determinarla a compiere o subire atti sessuali su o da una terza persona.

(8) Si applica la pena della reclusione non inferiore ad anni cinque qualora il colpevole

1. faccia uso, durante la commissione del fatto, di un 'arma o di altro oggetto pericoloso

2. la vittima, durante la commissione del fatto

a) subisca gravi maltrattamenti o

b) si venga a trovare in pericolo di vita.

(9) Nei casi di minore gravità degli Abss. 1 e 2 si applica la pena della reclusione da mesi 3 ad anni 3, nei casi di minore gravità degli Abss. 4 e 5 la reclusione da mesi 6 ad anni 10, nei casi di minore gravitá degli Abs. 7 e 8 si applica la pena della reclusione da anni 1 ad anni 10 .
(2) Alla stessa pena soggiace chi abusa di una persona incapace di resistere (ai sensi dell' Abs. 1) in modo tale da determinarla a compiere o subire atti sessuali su o da una terza persona.

(3) Nei casi di particolare gravità la pena non può essere inferiore ad anni 1 di reclusione.

(4) Il tentativo é punibile.

(5) La pena é della reclusione non inferiore ad anni 2 qualora

1. l'Autore si congiunga carnalmente con la vittima, o compia o faccia compiere su di sé analoghi atti sessuali, consistenti, in particolare, in una penetrazione del corpo della vittima (stupro),

2. più persone concorrano nella commissione del fatto,

3. l'autore ponga l'incapace, mediante la commissione del fatto, in grave pericolo di subire rilevanti danni alla salute o un consistente nocumento al normale sviluppo psichico o fisico.

(6) Nei casi di minore gravità dell'Abs. 5 si applica la pena della reclusione da anni 1 ad anni 10.

\section{IL SEXUALSTRAFRECHT TEDESCO PREVIGENTE}

Prima di procedere all'analisi dettagliata dei contenuti della legge di riforma della normativa penale sessuale tedesca, è d'obbligo fornire taluni approfondimenti relativi alla legislazione previgente in materia. Solo grazie alla conoscenza di quest'ultima, invero, è possibile valutare l'effettivo impatto innovativo, cogliere la ratio, e formulare ipotesi sulla concreta utilità ed efficacia (preventiva e repressiva) dell'intervento riformista de quo.

\subsection{L' evoluzione storica del diritto penale sessuale tedesco ante reforma}

Premettendo che non è possibile in questa sede occuparsi troppo diffusamente dello sviluppo storico del Sexualstrafrecht d'oltralpe ${ }^{19}$, si ritiene tuttavia opportuno suddividere la

\footnotetext{
${ }^{19}$ Al riguardo si rimanda nella letteratura tedesca, ex plurimis, a FROMMEL, Monika. Die Reform der Reform der Reform ...des Sexualstrafrechts - eine endlose Geschichte. NK, 2003, 46 p.; KIELER, Marita. Tatbestandsprobleme der sexuellen Nötigung, Vergewaltigung sowie des sexuellen Missbrauchs widerstandsunfähiger Personen, Berlin: Tenea, 2003. p. 13.; LAUBENTHAL, Klaus. Sexualstraftaten, Heidelberg: Springen, 2000, 3 p. Per quanto riguarda gli scritti in italiano, sia consentito il rinvio a MACRì, Francesco. Verso un nuovo diritto penale sessuale. Firenze: FUP, 2010. p. 53.
} 
trattazione in due parti, di cui la prima (A) relativa alla fase dal 1871 al 1997, e la seconda (B) concernente il periodo dal 1997 al 2016.

\subsubsection{Evoluzione storica del diritto penale sessuale tedesco dal 1871 al 1997}

Nel Codice penale per l'impero tedesco del 15 maggio 1871, i reati sessuali erano collocati tra $\mathrm{i}$ "Delitti e contravvenzioni contro la morale pubblica", la cui finalità primaria era la punizione delle "violazioni dell' ordine sessuale precostituito"20.

Nella disciplina penalistica vigente in Germania dal 1871 fino al 1973, il magistero penale interveniva in presenza di ogni rapporto sessuale che non fosse consumato nella monogamica e istituzionalizzata comunione di vita tra un uomo ed una donna (ergo non caratterizzato dalla finalità riproduttiva) ${ }^{21}$. Cartina di tornasole di tale vetusta concezione era la figura criminosa sessuale di maggiore rilevanza nel codice del 1871, cioè la "Violenza carnale" (“Notzucht”) di cui al § 177 RStGB, che includeva nel suo 'mirino incriminatore' unicamente $i$ rapporti sessuali consumati al di fuori del matrimonio 22 .

A livello tecnico-sistematico, $i$ due principali reati sessuali della codificazione germanica del 1871 erano la già citata "Violenza carnale” (§ 177 1. Alt. RStGB) e gli “Atti lussuriosi” (“Unzüchtigen Handlungen”, § 176 Nr. 1 RstGB): la violenza carnale all'epoca, come in molti altri ordinamenti, incriminava unicamente il coito vaginale completo ${ }^{23}$.

Sotto il profilo dei mezzi coercitivi rilevanti, l'illiceità penale era limitata alle sole aggressioni sessuali perpetrate mediante violenza o minaccia grave. Per quanto concerne il concetto di violenza, all'epoca lo stesso presupponeva una tenace e netta resistenza da parte del soggetto passivo, ed il superamento della medesima da parte dell'agente attraverso un massiccio impiego di forza fisica: un mero respingimento o una fiacca opposizione all'approccio

\footnotetext{
${ }^{20}$ WELZEL, Hans. Das deutsche Strafrecht, 2a Ed. Berlin: De Gruyter, 1949, p. 195.

${ }^{21}$ Cfr. KIELER, Marita. Tatbestandsprobleme der sexuellen Nötigung, cit., p. 15, la quale evidenzia come "il rapporto sessuale con finalità diverse da quella riproduttiva, era considerato, nell'immaginario collettivo persistente fino agli anni ' 60 del secolo scorso, come diretto a perseguire una 'sfrenata anelazione del piacere', e come espressione di edonistica e irrefrenabile lidibine".

${ }^{22}$ Sul punto v. SICK, Brigitte. Sexuelles Selbstbestimmungsrecht und Vergewaltigungsbegriff. Berlin: Duncker \& Humblot, 1993, p. 58.

${ }^{23}$ Ciò si giustificava, in tale contesto, in considerazione del fatto che tale tipo di interrelazione sessuale era vista come la naturale e piena soddisfazione edonistica degli istinti carnali, ed inoltre era l'unica condotta atta a provocare, eventualmente, la perdita della verginità e la gravidanza della vittima.
} 
non erano sufficienti, “essendo gli stessi spesso poco seri” (nel senso di non rappresentare un chiaro indice del dissenso della vittima) ${ }^{24}$.

La descritta legislazione penale sessuale si mantenne sostanzialmente immutata fino al secondo dopoguerra. A partire all'incirca dal 1960, i giuristi d'oltralpe iniziarono però ad esternare sempre più incisive critiche alla connotazione eticizzante e maschilista del diritto penale sessuale tedesco. Il grande movimento riformista innescato dalla rivoluzione sessuale portò infine all'approvazione di due importanti riforme penalistiche in materia sessuale. La prima si ebbe con la 1. Strafreformgesetz, dell'1 settembre 1969, la quale depenalizzò le interrelazioni sessuali omosessuali tra adulti maschi consenzienti, gli 'atti lussuriosi' con animali e la fattispecie criminosa di “Ottenimento fraudolento di rapporti sessuali extra-matrimoniali" 25 . Successivamente, con la 4. Strafreformgesetz del 23 novembre 1973, il legislatore tedesco incise in modo rilevante sui reati sessuali dello $\mathrm{StGB}^{26}$. Le principali innovazioni introdotte a mezzo del predetto strumento legislativo furono anzitutto la conversione dell'intitolazione del XIII. Titolo da "Reati contro la morale pubblica" a "Reati contro la libertà di autodeterminazione sessuale" ("Straftaten gegen die sexuelle Selbstbestimmung") 27. Si provvide, inoltre, a mutare radicalmente le espressioni deputate all' individuazione delle interrelazioni sessuali penalmente rilevanti: gli “atti lussuriosi” divennero perciò "atti sessuali” ("Sexuelle Handlungen”) e la "Violenza carnale" si trasformò in "Stupro" ("Vergewaltigung").

Pur riconoscendo i meriti di tale riforma sotto il profilo della modernizzazione e 'demoralizzazione' delle fattispecie, molti autori però ne criticarono al contempo la mancanza di coraggio, essendo rimasta praticamente immutata la struttura delle principali ipotesi criminose, cioè i delitti di "Stupro" e "Costrizione sessuale". Oggetto di strali critici furono inoltre il mantenimento dei mezzi costrittivi "violenza" e "minaccia grave" e della non punibilità delle aggressioni sessuali tra coniugi, e la circoscrizione dello stupro ai casi di penetrazione maschile della vagina femminile ${ }^{28}$.

Sulla base di quanto appena asserito, si comprende agevolmente come gli aneliti di (ulteriore) modifica del diritto penale sessuale d'oltralpe si mantennero nella società e

\footnotetext{
${ }^{24}$ OPPENHOFF, Friedrich. Das Strafgesetzbuch für das Deutsche Reich, 12a Ed. Berlin: De Gruyter, 1891, § 176, Anm. 11.

${ }^{25}$ Al riguardo, v. STRATENWERTH, Guenter; SCHULTZ, Hans. Leitprinzipien der Strafrechtsreform. Köln: Springer, 1970.

${ }^{26}$ La R di Reich [impero] era stata espunta dopo la trasformazione della Germania in Repubblica.

${ }^{27}$ KIELER, Marita. Tatbestandsprobleme der sexuellen Nötigung . cit., p. 18.

${ }^{28}$ FROMMEL, Monika. Das klägliche Ende der Reform der sexuellen Gewaltdelikte. ZRP, 1988, p. 164.
} 
comunità giuridica tedesche anche dopo il 1973, ed in particolare negli anni ' 80 e '90, quando rilevanti argomenti pro riforma vennero forniti da importanti studi ed analisi in materia sessuale delle scienze criminologiche, psicologiche e sociologiche, oltre che dalla crescita dei movimenti per la tutela dei diritti delle donne.

\subsubsection{Evoluzione storica del Sexualstrafrecht dalla riforma del 1997 a quella del 2016}

Gli aneliti di riforma appena menzionati si sono tradotti in ben tre interventi 'riformisti' del legislatore tedesco (rispettivamente datati 1997, 1998 e 2003), che in linea generale hanno perseguito una finalità di estensione della tutela penalistica di categorie soggettive particolarmente vulnerabili sotto il profilo sessuale ${ }^{29}$. Con le stesse novelle legislative si è proceduto, inoltre, ad una modernizzazione ed affrancamento del diritto penale sessuale da quegli appena menzionati vecchi stereotipi ormai intollerabili per una moderna sensibilità giuridica. In tal senso vanno lette l'eliminazione di ogni caratterizzazione di genere del soggetto passivo dei reati sessuali (ora indifferentemente uomo o donna), e l'abrogazione dell' inaccettabile incriminazione dei rapporti omosessuali consensuali con minorenne, in casi in cui i corrispondenti rapporti eterosessuali erano leciti.

Le innovazioni di maggior rilievo apportate da tali provvedimenti legislativi, e nello specifico dalla 33. Strafänderungsgesetz del 1. luglio 1997, sono consistite in primis nell'unificazione (sistematica ma non sanzionatoria) dei reati di "Stupro" e "Costrizione sessuale” nel nuovo § 177 StGB ("Costrizione sessuale; Stupro"). Si è altresi proceduto ad ampliare la portata applicativa dello stupro ben oltre gli angusti limiti della penetrazione dell'organo maschile nella vagina. La novità maggiormente significativa è stato però, con tutta probabilità, l'introduzione nel reato di “Costrizione sessuale; Stupro" di una nuova modalità costrittiva che si aggiunge alla tradizionali violenza e minaccia grave: la c.d. Ausnutzungsvariante ( $\$ 177$ Abs. $1 \mathrm{nr}$. 3). Con siffatta nuova previsione si sono ricondotte alla categorie delle aggressioni sessuali costrittive penalmente rilevanti anche quelle in cui l'agente realizzi le attività sessuali volute, "approfittando di una situazione nella quale la vittima si trova indifesa alla mercé dell'agente". Con essa il legislatore tedesco del 1997 ha inteso conseguire

29 Trattasi, rispettivamente, della 33. Strafrechtänderungsgesetz (StäG) del 1 luglio 1997, della 6. Strafrechtsreformgesetz del 1 aprile 1998, e infine della 35. StäG del 27 dicembre 2003. 
una tutela maggiormente incisiva della libertà sessuale colmando alcune 'lacune punitive' (Strafbarkeitsluecken) derivanti dalla dalla rigorosa prassi applicativa del BGH in tema di costrizione sessuale violenta e minacciosa (oggetto di approfondimento nei successivi paragrafi): è peraltro da rilevare che in ogni caso - omettendo (nel 1997, così come nel 1998 e poi nel 2003) una svolta 'consensualistica' tout court, il legislatore tedesco non abbia peraltro eliminato numerose altre 'lacune punitive', le quali avrebbero poi giustificato la riforma del 2016.

\subsection{La nozione cardine di "atto sessuale" ("Sexuelle Handlung") nel diritto penale sessuale tedesco anteriore al 10.11.2016}

La già citata riforma legislativa tedesca del 1973 ha tipizzato i reati contro l'autodeterminazione sessuale incentrandoli sulla nozione di "Sexuelle Handlungen" ("atti sessuali”), il cui compimento con, da, su o di fronte alla vittima è richiesto in pressoché tutti i reati sessuali dello StGB.

All'uopo, è stata inserita una norma definitoria, il $\S 184 \mathrm{f} \mathrm{StGB}$, al fine di orientare l'opera interpretativa del giudice nella selezioni dei comportamenti concretamente qualificabili come "atti sessuali". Tale norma dispone che "ai sensi di legge si intendono 'atti sessuali' solo quelli che, con riguardo ai beni giuridici rispettivamente tutelati [ndr: dalle varie fattispecie], siano di rilevante gravità".

Il $\S 184 \mathrm{f}$ del codice tedesco, in realtà, non contiene una vera e propria definizione di “atti sessuali”, bensì si limita a prescrivere che, tra essi, assumano rilevanza penale soltanto quelli di una certa gravità, adottando a tal fine il parametro del bene/oggetto giuridico specifico rispettivamente tutelato da ciascuna delle singole incriminazioni imperniate sugli "atti sessuali"30.

In tale contesto edittale, l'interprete deve dunque in primis determinare i criteri di individuazione della sessualità delle condotte umane, cioè quando si è effettivamente in presenza di un "atto sessuale" e quando invece no; ed in seconda battuta, identificare i parametri in base ai quali restringere ulteriormente la categoria di cui sopra, circoscrivendola agli "atti sessuali di rilievo": al riguardo occorrerà considerare lo specifico oggetto di tutela della singola fattispecie. Ai fini del presente commento, ci si limiterà peraltro ai delitti di “Costrizione sessuale/Stupro” ( $\$ 177$ StGB) e di “Abuso sessuale” ( $\$ 179$ StGB), considerando che

${ }^{30} \mathrm{~V}$. ad esempio in tal senso LAUBENTHAL, Klaus. Sexualstraftaten, cit., p. 19. 
gli stessi - entrambi 'fusi' nel nuovo § 177 - sono stati incisivamente modificati dalla Riforma del $2016^{31}$.

Iniziando dagli orientamenti dottrinali e giurisprudenziali sui criteri di individuazione della natura sessuale "generica" di un atto, è da rilevare come sussista un generale consenso sul fatto che - eliminato ogni riferimento legislativo a concetti 'soggettivamente orientati' come quello di "atti lascivi" - una condotta, per essere qualificata "atto sessuale" debba presentare già nella sua materialità una connotazione di carattere sessuale.

Va però sottolineato che né nelle sentenze della Corte Suprema tedesca (Bundesgerichtshof, abbreviato $\mathrm{BGH}$ ), né soprattutto nelle opere dottrinali, si rinvengono costruzioni ermeneutiche di carattere generale dirette a specificare in cosa consista la “connotazione sessuale oggettiva” (“objektiver Sexualbezug”) della condotta32.

Vi sono comunque delle 'linee guida', accolte sia in dottrina che in giurisprudenza, per circoscrivere il novero degli atti sessuali (salvo poi individuare tra di essi quelli più gravi ex $\S$ $184 \mathrm{f} \mathrm{StGB})$. Un primo punto fermo è che, se come già visto l'intento sessuale soggettivo dell'agente non è sufficiente per connotare sessualmente una condotta che tale non è oggettivamente, tale intento non è altresì necessario, dovendo qualificarsi come "atti sessuali" anche comportamenti determinati esclusivamente, nell'agente, da finalità affatto distinte (es. scherzo, vendetta ecc. $)^{33}$.

Si é poi introdotto un ulteriore parametro di valutazione per discernere il carattere sessuale o meno delle condotte ambivalenti, cioè quelle (per lo più ricadenti su zone genitali, mammellari o prossime alle stesse) le quali in astratto possono rivestire o meno significato sessuali, come segnatamente gli atti compiuti nel corso di visite mediche (es. penetrazione di un dito in vagina da parte del ginecologo).

In definitiva, da un'analisi complessiva degli orientamenti dei giuristi tedeschi sul concetto di "atti sessuali", non emerge chiaramente, stante la genericità e non univocità dei parametri individuati, l'effettiva estensione applicativa del concetto in esame. Ciò constatato,

\footnotetext{
${ }^{31}$ Che ha altresì inserito un nuovo delitto di "Molestie sessuali" ex § 184i StGB: al riguardo sarà dunque interessante capire - nei successivi paragrafi - gli eventuali criteri di differenziazione tra gli atti di molestia sessuale previsti da tale ultima previsione, e gli "atti sessuali" di cui al nuovo (e vecchio) § 177 StGB.

32 (Il BGH= Bundesgerichtshof è l'equivalente tedesco della Corte di Cassazione italiana e del Supremo Tribunal Federal brasiliano). In tal senso BGHSt. 29, p. 338; in dottrina, ex plurimis, LENCKNER, Theodor. § 184f StGB. In: SCHÖNKE, Adolf; SCHRÖDER, Hoerst; LENCKNER Theodor. Strafgesetzbuch Kommentar, 27a Ed. München: Beck, 2006. 6 Rn.; SICK, Brigitte. Sexuelles Selbstbestimmungsrecht, cit., p. 258.

${ }^{33}$ GÖSSEL, Karl Heinz. Das neue Sexualsfrafrecht. Berlin: De Gruyter, 2005, p. 17.
} 
si rende necessario esaminare in concreto quali tipologie di comportamenti umani sono stati identificati come "Sexuelle Handlungen" da dottrina e, in particolare, giurisprudenza: sempre tenendo presente che ai fini della concreta rilevanza penale occorre anche una certa gravità, oltre che la sessualità, della condotta.

Chiaramente, non vi sono mai state discussioni sul carattere sessuale della penetrazione vaginale, orale od anale, anche attuata mediante oggetti, e sussiste un generale consenso sulla sessualità dei toccamenti degli organi genitali, del seno nella donna e dei loro contorni, e dell'imposizione non gradita di baci con la lingua ${ }^{34}$. Si è ulteriormente precisato come non sia rilevante, in caso di zone genitali o mammellari e dei loro contorni, la durata del toccamento, il fatto che quest' ultima avvenga al nudo o con l'intermediazione di vestiti e la delicatezza o meno dello stesso ${ }^{35}$. Non si riscontra invece la natura sessuale negli atti di toccamento del torace (negli uomini), delle spalle, delle gambe, dei capelli, e di regola anche nei "baci e abbracci consueti" (come quelli che ci si scambia al momento di salutarsi), anche se ricadenti su zone che in relazione al singolo soggetto possono classificarsi come 'erogene' ${ }^{36}$.

Alla luce di tutto ciò, emerge senz'altro nei giuristi teutonici una evidente tendenza a prediligere una valutazione di fatto 'anatomica' delle condotte ai fini della loro qualificazione come "Sexuelle Handlungen".

Una volta accertato il carattere sessuale degli atti posti in essere dall'agente ciò non è ancora sufficiente per sancirne la rilevanza penale sessuale ai sensi dello StGB, dovendosi 'superare' la soglia di gravità (“Erheblichkeitsschwelle”) prevista dal § 184f StGB.

Pur non potendo trattare esaustivamente della complessa questione ${ }^{37}$, preme osservare come la giurisprudenza, tedesca abbia affermato la non gravità / rilevanza ex $\S 184 \mathrm{f}$ StGB di taluni atti valutati di "significato sessuale" modesto: trattasi di toccamenti molto brevi, o comunque di scarsa lesività sessuale come fugaci strette per i fianchi o baci sulle guance ${ }^{38}$.

\subsection{I mezzi di costrizione 'tradizionali' del Sexualstrafrecht previgente: violenza e minaccia grave.}

\footnotetext{
${ }^{34}$ WOLTER Juergen; HORN Eckhard. \$ 184f StGB. In: RUDOLPHI, Hans Joachim; HORN, Eckhard; SAMSON, Erich. SK - StGB. 68a Ed. Muenchen: Beck, 2007. 6 rn.

${ }^{35}$ In tal senso WOLTER Juergen; HORN Eckhard. \$ $184 \mathrm{f} \mathrm{StGB,} \mathrm{cit.} 6 \mathrm{rn}$. In giurisprudenza cfr. BGH. StV, 2000, p. 197.

${ }^{36}$ LAUBENTHAL, Klaus. Sexualstraftaten, cit., p. 20.

${ }^{37}$ All'uopo si rimanda agli scritti degli autorevoli autori citati nelle precedenti note.

${ }^{38}$ Tra le tante v. BGH. NstZt. 1983. 553 p.; BGH. StrVert.1983. 415 p. In dottrina v. GÖSSEL, Karl Heinz. Das neue Sexualsfrafrecht, cit., p. 19.
} 
Il reato sessuale 'centrale' (sotto alcuni profili equivalente, affiancato dal § 179 StGB per gli abusi sessuali - alla nostra "Violenza sessuale" ex art. 609-bis c.p.) del Testo punitivo tedesco era - ed è ancor più, sebbene con incisive modifiche, dopo la riforma del 2016 - quello di “Costrizione sessuale / Stupro” di cui al § 177 StGB. Tale fattispecie, all'Abs. 1, ha contemplato dal 1997 al 2016 tre distinte modalità di commissione degli atti sessuali, date da:

A) Violenza.

B) Minaccia grave ("di un pericolo attuale per la vita o per l'incolumità fisica”).

C) "Approfittamento di una situazione in cui la vittima è indifesa alla mercé dell'agente" (mezzo di costrizione introdotto dalla riforma del 1997),

Riservando al paragrafo successivo l'analisi di tale ultima modalità, occorre innanzitutto approfondire la dottrina, e soprattutto la giurisprudenza, tedesca sui due mezzi di imposizione 'tradizionali' degli atti sessuali, cioè la violenza e la minaccia grave, in modo tale da cogliere l'effettiva ampiezza di quelle 'lacune della punibilità', la presenza delle quali ha determinato la recente riforma commentata nel presente scritto.

A) Violenza.

La maggioranza delle pronunce del tribunale tedesco di ultima istanza in materia penale, il Bundesgerichtshof $(B G H)$, concernenti la disposizione di "Costrizione sessuale; Stupro" riguardano - così come avviene nella giurisprudenza italiana ${ }^{39}$ - aggressioni sessuali commesse mediante violenza ${ }^{40}$.

Per violenza, ai sensi del $\S 177 / 1$ n. 1 StGB previgente, nella giurisprudenza di legittimità dell'Alta Corte tedesca, si è inteso l'impiego di forza fisica - avente un influsso diretto sul corpo della vittima e da quest'ultima avvertito come coartazione fisica - legato da un nesso eziologico/strumentale con il successivo o contemporaneo compimento di atti sessuali ${ }^{41}$.

Sulla base di tale orientamento, di cui è evidente la netta differenziazione rispetto a quello accolto dalla Cassazione italiana sin dagli anni '90, e coincidente con "qualunque condotta diretta a coartare l'altrui volontà di non compiere o subire atti sessuali"42, il novero delle condotte sessualmente 'violente' è stato circoscritto sotto molteplici profili.

\footnotetext{
${ }^{39}$ Sia consentito al riguardo il rinvio a MACRì, La violenza sessuale (art. 609-bis c.p.) nella giurisprudenza della Suprema Corte del 2015. Diritto penale contemporaneo (rivista trimestrale), ${ }^{\circ} 1,2016$, p. 162.

${ }^{40}$ In questa sede intesa unicamente come 'violenza fisica' (e non anche psichica, inclusa nella 'minaccia').

${ }^{41}$ V., ex plurimis, BGH, 1 luglio 1981 - 3 StR 151/81. NJW, 1981. 2204 p.; BGH, 13 aprile 1999 - 4 StR 101/99. NstZ, 1999, p. 506.

${ }^{42}$ Cass., sez. III, 30 marzo 2000, n. 1405, Delle Donne. Giust. Pen. 2000, p. 685.
} 
In primo luogo, sono state qualificate "non violente" condotte di natura verbale o meramente ingannatoria come "molestie, importunamenti, impertinenze e anche la mera conduzione, mediante raggiro, della vittima in un posto contro la sua volontà" ${ }^{43}$.

Il BGH poi, sulla base anche di una interpretazione sistematica scaturente dal fatto che la minaccia costrittiva è limitata ai casi più gravi ${ }^{44}$, pur non richiedendo un massiccio impiego di forza fisica ${ }^{45}$, ha peraltro affermato che occorra un influsso corporeo di una certa rilevanza ${ }^{46}$, sulla cui misura minima non è stato peraltro fissato alcuna parametro uniforme ${ }^{47}$. Si è comunque escluso che costituisca violenza una 'mera spinta sul divano'. ${ }^{48}$

Sotto il profilo della strumentalità della violenza rispetto al compimento degli atti sessuali il BGH si è altresì attestata su posizioni restrittive, escludendo la rilevanza ai sensi del § $177 / 1$, n. 1 previgente delle aggressioni repentine o a sorpresa ("überraschende Sexualangriffe”). La giurisprudenza teutonica ha affermato, al riguardo ${ }^{49}$, come in tali casi la vittima non abbia il tempo di maturare un effettivo dissenso, e pertanto la repentina aggressione non possa configurarsi come una violenza tale da sopraffare una contraria volontà: il nesso di strumentalità tra violenza e atti sessuali verrebbe infatti meno, coincidendo la violenza col patimento stesso dell'atto da parte della vittima (trattandosi dunque non più di violenzamezzo, bensì di violenza-fine $)^{50}$.

B) Minaccia.

Al riguardo va innanzitutto osservato come il BGH abbia ricostruito il "pericolo attuale per l'incolumità fisica' - cui è subordinata la rilevanza della minaccia ex § 177/1, n. 2 StGB previgente - in senso restrittivo, non ritenendo lo stesso sussistente ove si minacci una lesione di lieve entità', come ad esempio il 'prendere la vittima a schiaffi' ${ }^{52}$ : casi in cui, peraltro,

\footnotetext{
43 BGH, 9 marzo 1990 - 3 StR 58/90. NstZ, 1990, p. 335.

${ }^{44} \mathrm{~V}$. nota precedente.

${ }^{45}$ WOLTERS Juergen; HORN, Eckhard. \$ 177 StGB, cit. 10 Rn.

${ }^{46}$ BGH, 13 aprile 1999 - 4 StR 101/99, cit.

47 FOLKERS, Susanne. Ausgewaehlte Probleme bei sexueller Noetigung und Vergewaltigung aus der Sicht der Praxis. Baden Baden: Nomos, 2004, p. 43.

48 BGH, 28 febbraio 1991 - 4 StR 553/90. BGHR, StGB, § 177, Abs. 1, Gewalt, p. 8.

${ }^{49}$ BGH, 2 giugno 1982 - 2 StR 669/81. NJW, 1982, 2264 p.; BGH, 22 aprile 1997 - 4 StR 140/97. NstZ, 1998, p. 133.

${ }^{50} \mathrm{Si}$ tenga presente, a tal riguardo, l'orientamento diametralmente opposto della Corte di Cassazione italiana, che qualifica pacificamente 'violenti' gli atti sessuali a sopresa: v., ex multis, Cass., Sez. III, 1 febbraio 2006, n. 6340, G. CED Cass. Pen., 233315.

51 Tra le pronunce più rilevanti v. BGH, 17 ottobre 2000 - 1 StR 270/00. NstZ, 2001. 246 p.; BGH, 8 maggio 2001 - 4 StR 58/01. StV, 2001, p. 679.

52 BGH, 13 dicembre 1990 - 4 StR 505/90. NstE, \& 177 StGB. 24 Nr.
} 
l'avveramento della minaccia darebbe luogo a costrizione sessuale violenta rilevante ex $\$ 177 / 1$, n. $1^{53}$.

E' tuttavia la problematica della 'minaccia concludente' (konkludente Drohung) ad aver spesso assunto particolare rilievo in tale contesto: per minaccia concludente s'intende, infatti, la prospettazione di un male - nell'ordinamento tedesco quanto meno la lesione rilevante dell'incolumità fisica - ingiusto, e dipendente dalla volontà dell'agente, non attraverso la comunicazione verbale, bensì mediante un comportamento univocamente espressivo (concludente appunto) $)^{54}$.

Parte della dottrina tedesca ha pertanto proposto un'applicazione estensiva della minaccia concludente quale possibile rimedio alla restrittiva interpretazione del requisito della violenza costrittiva di cui al $\S 177 / 1$, n. 1 StGB previgente ${ }^{55}$. La giurisprudenza non ha però mostrato la medesima inclinazione, delimitando il ricorso alla figura in questione alla sussistenza di tassativi presupposti, spesso non riscontrabili in molti casi concreti di costrizione sessuale ${ }^{56}$.

E' evidente, dunque, come gran parte delle aggressioni sessuali poste in essere in contesti di intimidazione ambientale o di violenze reiterate (spesso ricorrenti nelle relazioni familiari o sentimentali) sfuggissero all'applicazione della minaccia (concludente) ex $\S 177 / 1, n$. $2 \mathrm{StGB}$, atteso che il nesso strumentale tra violenze (precedenti) e approccio sessuale (attuale) sovente si sostanzia in un' efficacia terrorizzante di natura prettamente psicologico-soggettiva.

\subsection{La nuova modalità costrittiva, c.d. Ausnutzungsvariante, introdotta nel 1997}

Quanto appena detta sull'esegesi 'restrittiva accolta dalla giurisprudenza tedesca in tema di costrizione sessuale violenta o minacciosa ex $\$ 177 / 1$, nn. 1 e 2 StGB previgente, rende chiara la ratio dell'introduzione nel 1997, per mezzo della 33. StAeG di un'ulteriore 'variante' costrittiva (c.d. Ausnutzungsvariante), data dal "approfittare di una situazione nella quale la vittima si trova indifesa alla mercé dell'agente".

\footnotetext{
53 SICK, Brigitte. Sexuelles Selbstbestimmungsrecht, cit., p. 201.

54 Esempi paradigmatici sono le rapine compiute - senza proferire parole minacciose - mediante ostensione o puntamento contro la vittima di armi da sparo o da taglio.

55 In tal senso HELMKEN, Dierk. Vergewaltigungsreform und kein Ende?. ZRP, 1995. 302 p.; SCHROEDER, Friedrich-Christian. Die Revolution des Sexualstrafrechts 1992 - 1998. JZ, 1999, p. 827.

${ }^{56}$ V. ad esempio BGH, 7 novembre 2002 - 3 StR 274/02. NStZ, 2003, p. 424.
} 
Il legislatore tedesco dell'epoca - in analogia con quello del 2016 - intendeva dunque conseguire una tutela maggiormente incisiva della libertà sessuale colmando all'uopo talune ‘lacune punitive’ derivanti dalle prassi giurisprudenziali appena esaminate.

A sostegno dell'utilità - e dell'equiparazione sanzionatoria alle aggressioni sessuali violente o minacciose - della nuova modalità di costrizione sessuale di cui al previgente $§ 177 / 1$, n. $3 \mathrm{StGB}$, si è fatto riferimento a una pluralità di autorevoli ricerche di psicologi, sociologi, criminologi e vittimologi. Tali indagini hanno dimostrato che spesso, di fronte ad approcci sessuali in contesti ambientali o relazionali coartanti, le vittime tendano ad essere paralizzate, non riuscendo in molti casi a reagire neanche verbalmente all'aggressione ${ }^{57}$.

Iniziando dall'esegesi del concetto di condizione di minorata difesa ("situazione nella quale la vittima è indifesa"), esso non ha dato luogo a divergenze, essendosi accolta l'elaborazione relativa all'affine concetto di cui all'abrogato $\$ 237$ StGB 58, ravvisandosi 'minorata difesa' in situazioni nelle quali le possibilità di difesa della vittima siano ridotte in misura tale da non costituire ostacoli rilevanti all'aggressione dell'agente ${ }^{59}$. Onde valutare la concreta sussistenza delle circostanze 'sintomatiche' di minorata difesa si è poi proposto in dottrina - in assenza di una chiara presa di posizione del BGH al riguardo, e in ossequio alla ratio di tutela della persona offesa che contrassegna l'Ausnutzungsvariante - di attribuire rilievo dirimente alla percezione soggettiva della situazione da parte della vittima piuttosto che a un criterio di valutazione ex ante su basi oggettive: questo per evitare facili elusioni della norma da parte di chi strumentalizzi coscientemente l'errore della vittima (sull'effettiva sussistenza di possibilità difensive) per costringere la stessa agli atti sessuali ${ }^{60}$.

Per quanto concerne l'ulteriore elemento essenziale della fattispecie, dato dall'approfittamento della situazione di minorata difesa da parte del soggetto agente, la giurisprudenza ha statuito come occorra in primo luogo che la particolare situazione in cui versi

\footnotetext{
${ }^{57}$ Ex multis HARBECK, Birgit. Probleme des Einheitstatbestandes sexueller Nötigung/ Vergewaltigung. Baden Baden: Nomos, 2001, p. 101.

${ }^{58} \mathrm{La}$ fattispecie incriminatrice suddetta, che incriminava il rapimento a scopo sessuale con la reclusione fino a 5 anni, prevedeva tra i suoi requisiti che la vittima venisse posta in una condizione hilflos (che indica letteralmente l'assenza di possibilità di aiuto/soccorso), del tutto analoga alla situazione schutzlos (che indica l'assenza di possibilità di protezione) di cui al previgente §177/1, n. 3 StGB.

59 Così BGH, 26 agosto 2005 - 3 StR 260/05. NStZ, 2006. 165 p. Esemplificando alcune circostanze ravvisate nella prassi, si pensi alla conduzione della vittima in un luogo isolato in cui la stessa non può sperare nella presenza di terze persone, alla compresenza di autore e vittima in un luogo chiuso privo di vie di fuga; e da ultimo all'età della vittima, alla sua stanchezza fisica o alla superiorità fisica dell'agente.

60 Tra i tanti LAUBENTHAL, Klaus. Sexualstraftaten, cit. 150 p.; contra MILDERBERGER, Elke. Schutzlos Hilflos - Widerstandsunfähig: Einige Anmerkungen zur Auslegung der Tatbestandserweiterung des § 177 StGB n. F. Muenster: LIT, p. 62.
} 
la vittima obiettivamente consenta o, quanto meno, agevoli la costrizione agli atti sessuali; e che inoltre sussista in capo all'agente la consapevolezza di tale condizione di minorata difesa, unita alla volontaria strumentalizzazione della stessa a fini sessuali ${ }^{61}$.

\section{LA DISCIPLINA VIGENTE DEL SEXUALSTRAFRECHT QUALE RISULTANTE DALLE RIFORMA DEL 2016}

Una volta descritte le caratteristiche principali del diritto penale sessuale tedesco vigente fino al 9 novembre 2016, è d'obbligo quindi approfondire i singoli contenuti della 50. legge di modifica al codice penale tedesco, la cui effettiva incisività potrà dunque essere colta dal confronto con le norme previgenti appena analizzate (e il cui testo è stato comunque riportato nella tabella n. 1).

Si precisa sin d'ora, ad ogni modo, che alcune disposizioni - per lo più di diritto processuale - della legge in esame non saranno in questa sede oggetto di analisi.

\subsection{La centralità del dissenso nella fattispecie 'base' di aggressione sessuale del §}

\section{$177 / 1$ StGB}

Come già accennato in precedenza, nel nuovo Sexualstrafrecht il delitto di cui al § 177 StGB riveste un ruolo assolutamente centrale, essendo state ricollocate in esso tutte le ipotesi di abuso sessuale prima incriminate dal $\S 179$. L'attuale $\S 177$, inoltre, è stato altresì arricchito, rispetto al precedente, da nuove fattispecie incriminatrici; e ne è inoltre state modificato l'apparato sanzionatorio.

In tale contesto, il delitto "simbolo" della presente riforma è quello definibile "Atti sessuali 'dissensuali' ", di cui al § 177/1 StGB, in virtù del quale sono punibili con la reclusione da 6 mesi a 5 anni gli atti sessuali realizzati “contro la volontà riconoscibile di un'altra persona". Trattasi della fattispecie 'base', deputata a tutelare le vittime dalle aggressioni sessuali meno gravi (seppur più gravi delle molestie sessuali, incriminate dal nuovo § 184i), posto che l'utilizzo di violenza o minaccia grave, così come la realizzazione di atti sessuali penetrativi ("Stupro") sono incriminati da autonome ipotesi criminose - tipizzate nei commi (Absätze) successivi - più severamente sanzionate.

${ }^{61}$ BGH, 26 agosto 2005 - 3 StR 260/05, cit. 
Il reato de quo, peraltro, esprime al massimo la carica simbolica e ideologica alla base dell'intervento di riforma, racchiusa nello slogan "No significa no" ("Nein heißt Nein") ampiamente utilizzato dagli organi mediatici per celebrare il nuovo diritto penale sessuale tedesco. É tuttavia curioso il fatto che tale incriminazione non fosse prevista dal progetto di legge governativo $18 / 8210^{62}$, e che il suo inserimento sia avvenuto per recepire le raccomandazioni del Bundesrat (Camera dei Länder) ${ }^{63}$.

La figura criminosa in esame, in connubio con quelle - 'sanzionatoriamente' equiparate del $§ 177 / 2$, e soprattutto con quella degli atti sessuali repentini/a sorpresa, esprime nel modo più evidente lo sforzo del legislatore di rimediare alla già analizzata giurisprudenza del BGH che - in relazione al previgente $§ 177$ - non puniva le aggressioni sessuali perpetrate con violenza o minaccia lievi, e ancor meno meramente dissensuali (Ausnutzungsvariante esclusa). Secondo quanto rilevato nella stessa relazione governativa, difatti, la pena minima all'epoca prevista (1 anno di reclusione), spingeva i giudici ad un certo self-restraint in fase applicativa: in virtù di ciò, il legislatore riformista ha configurato una pena minima di 6 mesi di reclusione per le succitate nuove fattispecie, mantenendo invece sostanzialmente le più severe pene di cui al vecchio § 177 per le ipotesi caratterizzate da violenza, penetrazione ecc.

Per quanto riguarda i profili sanzionatori, non può non rilevarsi come il minimo edittale di 6 mesi di reclusione (§§ 177/1 e 177/2 StGB) appaia eccessivamente mite, e altresì come l'elevazione del massimo edittale dai 5 anni delle suddette norme ai 15 anni dei $\S \S 177 / 4$ - 177/8 appaia alquanto incongrua: più condivisibile sarebbe stato prevedere un compasso edittale di 1 - 7 anni per i reati di cui ai $\S \S 177 / 1$ e 177/2, e una forbice tra 2 (invece di 1) e 15 anni di reclusione per le fattispecie aggravate di cui ai $\$ \$ 177 / 4$ e 177/5 StGB (e ovviamente minimi edittali rispettivi di 3, 4, e 6 anni per le incriminazioni di cui ai §§ 177/6, 177/7 e 177/8).

Tornando invece a focalizzarci sul \$177/1 (“Atti sessuali 'dissensuali' ”), le maggiori criticità, sia a livello applicativo che ermeneutico, scaturiscono senz'altro dall'espressione "Compiere atti sessuali (...) contro la volontà riconoscibile di un'altra persona". Il concetto di "volontà riconoscibile" è invero del tutto inedito non solo per il Sexualstrafrecht tedesco, ma altresì per tutte le legislazioni penali sessuali dell'Europa continentale. Lo stesso sembrerebbe avvicinarsi a quello di "mancanza di consenso" ("Lack of Consent") su cui si imperniano i reati

\footnotetext{
62 Il testo e la relazione di accompagnamento di tale Gesetzentwurf sono consultabilionline (in formato pdf) all'indirizzo <http://dip21.bundestag.de/dip21/btd/18/082/1808210.pdf >.

63 Il testo integrale contenente le raccomandazioni e osservazioni del Bundesrat è consultabile all'indirizzo web < http://dip21.bundestag.de/dip21/btd/18/086/1808626.pdf $>$.
} 
sessuali - a partire dallo "Stupro" ("Rape") - negli ordinamenti di Common Law": l'accostamento, peraltro è da limitare alla mera valorizzazione del consenso, posta la diversità ontologica tra il 'riconoscibile dissenso' e la 'mancanza di consenso', includendo la seconda anche casi di evidente assenza del dissenso (es.: atti sessuali repentini, su persona incosciente ecc.) ${ }^{65}$. Per quanto riguardo la prescrizione che il dissenso sia 'riconoscibile', la stessa è motivata - da quanto si legge nella stessa relazione al progetto di legge - dalla necessità di non fare dipendere totalmente la tipicità degli atti sessuali, in assenza di qualunque esternazione del medesimo, da un mero state of mind interiore del soggetto passivo

Andando oltre, due sono le maggiori critiche rivolte da taluni giuristi teutonici alla scelta legislativa di impiegare l'espressione "volontà riconoscibile":

1) Si è asserito che una tale formulazione della norma comporti il rischio di punire de facto condotte colpose con la pena riservata ad aggressioni sessuali dolose, qualora l'agente, in buona fede, non percepisca la "riconoscibile volontà" contraria della vittima ${ }^{66}$. A ciò si è tuttavia obiettato che comunque il giudice dovrà accertare - ai sensi dei principi generali - il dolo dell'agente relativamente a tutti gli elementi della fattispecie, e che comunque la "buona fede" di quest'ultimo andrà sottoposta ad un attento vaglio processuale ${ }^{67}$.

2) In stretta correlazione con la precedente critica, si è affermato che serie criticità - in punto di determinatezza - pone la determinazione del criterio in base al quale giudicare la "volontà riconoscibile": la volontà dovrà essere riconoscibile secondo il parametro di valutazione del soggetto attivo, del soggetto passivo o di un cittadino ragionevole ${ }^{68}$. Al riguardo viene peraltro segnalato il rischio, ulteriore rispetto a quello di condanne per aggressioni sessuali in concreto "colpose", del permanere - se non dell'allargarsi - di quelle lacune di tutela penalistica delle vittime più volte menzionate e la cui eliminazione ha costituito la ratio della presente riforma: ciò, ad esempio, ove ci si focalizzasse sul soggetto attivo - e magari sulla sua insufficiente conoscenza della psicologia e delle forme di comunicazione (verbali e non-verbali)

\footnotetext{
${ }^{64}$ V. ad es. la S. 1 del Sexual Offences Act 2003 inglese, su $<$ www.legislation.gov.uk/ukpga/2003/42/section/1>.

65 Sia consentito menzionare come in MACRì, Francesco. Verso un nuovo diritto penale sessuale, cit., vengano prospettati (p. 9) tre distinti modelli generali di disciplina dei reati sessuali: "vincolato", "consensuale limitato" e "consensuale puro". Il modello prescelto dal legislatore tedesco per il § $177 / 1$ StGB appare dunque chiaramente quello "consensuale limitato", che tra l'altro - per meglio differenziare gli "abusi sessuali" dalle "aggressioni sessuali" - era stato prescelto altresì dallo scrivente nella progetto di legge inserito al termine dello scritto (p. 159).

${ }^{66}$ FISCHER, Thomas. Zum letzen Mal: Nein heißt Nein, cit.

${ }^{67}$ HÖRNLE, Tatjana. Kopfschüitteln oder Weinen genügt, cit.

68 In tal senso MÜLLER, Henning Ernst. Der “erkennbare” Wille, cit.
} 
del dissenso/consenso sessuale femminile - quale parametro di valutazione della "riconoscibilità" del dissenso sessuale della vittima.

Tali critiche, ad ogni modo, appaiono ispirate ad una visione eccessivamente 'dogmatico-sacrale' del principio di legalità nel campo del diritto penale sessuale. É infatti da rilevare come anche per gli atti sessuali violenti, minacciosi ecc. si ponga un simile problema, relativo all'erronea supposizione del consenso: in Italia, ma altresì in Germania, il consenso dell'avente diritto - in relazione agli atti sessuali - opera come scriminante degli atti sessuali violenti ecc. ${ }^{69}$, ed ove l'agente supponga il consenso della vittima per errore determinato dal colpa, questi ugualmente andrà esente da pena, non essendo contemplate al momento - in nessun ordinamento di Civil Law - ipotesi colpose di reati sessuali ${ }^{70}$. Al momento, peraltro, dall'analisi massiccia delle sentenze emesse, quantomeno in Italia, dalla Suprema Corte, risulta la sostanziale assenza, nella prassi applicativa, di sentenze in cui l'imputato venga assolto in quanto supponente erroneamente un consenso agli atti sessuali in realtà assente, essendo l'eventuale esito favorevole per l'autore di regola determinato dalla 'comprovata' (tenendo presenti le regole sull'onore della prova nel processo penale) natura consensuale un'interrelazione sessuale. Il problema, peraltro, è ineludibile qualunque sia il modello di disciplina del diritto penale sessuale adottato, come dimostrato dall'infinito dibattito che nel mondo anglosassone caratterizza la defence dell'erronea supposizione del consenso tra tesi soggettiva, che richiede la sola 'onesta' ed effettiva convinzione della sussistenza del consenso, e tesi oggettiva, per la quale occorre altresi la ragionevolezza, valutata sul parametro della reasonable person, dello stesso ${ }^{71}$. Il problema, in realtà, è immanente al tipo di condotte considerate, e dipende dal fatto che - in assenza, come spesso accade, di prove di violenza massiccia $^{72}$ - il consenso della vittima, o l'erronea supposizione dello stesso, non può essere a priori escluso, e deve essere sempre oggetto di attento vaglio processuale.

In definitiva, pertanto, è da rilevare come il § 177/1 StGB stabilisca chiaramente il principio che ogni atto sessuale contrastante con la volontà della vittima - espressa o non

\footnotetext{
${ }^{69}$ Salvo che la violenza assurga a lesioni di gravissima entità, ma in tal caso si applicheranno - almeno in Italia - le fattispecie a tutela della vita e dell'incolumità fisica, artt. 577 ss. c.p. Italiano.

70 Figure criminose che invece, ad avviso dello scrivente, andrebbero introdotte per garantire una maggiore tutela delle vittime, ed una più equa e calibrata reazione sanzionatoria nei confronti degli autori. V. MACRì, Francesco. Verso un nuovo diritto penale sessuale, cit., p. 181.

${ }^{71}$ Al riguardo v. PAVARANI, Cristina. Il mero dissenso della vittima nella violenza sessuale: profili di diritto italiano e anglosassone. Ind. pen., II, 2002. 771 p. Nella dottrina britannica v., ex multis, CARD, Richard; GILLESPIE, Alisdair; HIRST, Michael. Sexual Offences, Bristol: Jordan, 2008, p. 72.

${ }^{72} \mathrm{Ma}$ anche in tale caso, l'agente potrebbe - e ciò in effetti si verifica sovente nella prassi - sostenere il consenso della vittima, ad esempio in un contesto di sessualità sado-masochista condivisa.
} 
espressa, ma comunque riconoscibile - costituisce una condotta penalmente rilevante: da ciò deriva la evidente conseguenza che siffatta norma penale svolge senz'altro altresì una funzione simbolica, ma ad avviso dello scrivente la stessa è - a differenza di altri casi - assolutamente condivisibile e congrua.

\subsection{Le nuove figure criminose di abuso sessuale, ed equiparate, ex $\S 177 / 2$ StGB}

Ulteriore norma dal pregnante contenuto innovatore rispetto al previgente assetto penale sessuale tedesco è il § 177/2 StGB, in seno al quale sono tipizzate ben 5 distinte figure criminose, alle quali è ricollegata la medesima (e - come detto - eccessivamente blanda) pena da 6 mesi a 5 anni di reclusione con cui sono sanzionati gli atti sessuali 'dissensuali' di cui al § $177 / 1$.

In tale disposizione sono peraltro accomunate ipotesi di 'abuso sessuale' nelle quali il destinatario dell'approccio non sia in grado di formare o esprimere la volontà contraria a causa di una situazione tendenzialmente (n. 1) o necessariamente (n. 2) di inferiorità fisica o psichica, casi nei quali il dissenso non possa inizialmente manifestarsi per la repentinità della condotta dell'agente (n. 3), ed infine ipotesi in cui la contraria volontà non sia manifestata a causa di una 'costrizione ambientale' lieve (n. 4) o di una diretta minaccia, seppur non grave (n. 5).

\subsubsection{Gli abusi delle condizioni di inferiorità fisica o psichica (\$ 177/2, nn. 1 e 2} StGB)

I nn. 1 e 2 del $§ 177 / 2$ StGB ricollegano la medesima pena del $\S 177 / 1$ (da 6 mesi a 5 anni) agli atti sessuali commessi:

1) Approfittandosi di una situazione in cui che tale persona non sia in grado di formare o esprimere una volontà contraria (n. 1); oppure

2) Abusando di una situazione in cui la capacità di formare o esprimere la volontà dell'altra persona, a causa della condizione fisica o psichica di quest'ultima, sia fortemente limitata (presupponendone il consenso agli atti sessuali).

Le due previsioni, pur nella differente formula, vanno analizzate congiuntamente, posto che entrambi configurano ipotesi di abuso sessuale, in cui l'agente strumentalizza a fini sessuali una inferiorità psichica o fisica della vittima: la stessa in realtà non è contemplata dalla lettera 
del n. 1), ma logicamente non è dato di immaginare casi in cui la persona "non sia in grado di formare o esprimere una volontà contraria" per motivi diversi da una condizione fisica o psichica. Si potrebbe all'uopo pensare ad ipotesi di “costrizione ambientale", come un approccio sessuale condotto - senza né minaccia, né violenza - da un aggressore robusto nei confronti di una ragazza esile in una zona completamente isolata in tempo notturno, ma in realtà tali ipotesi sono già adeguatamente coperte da due altre disposizioni: il $\S 177 / 2$, n. 4), per i casi meno gravi, e la fattispecie aggravata del $\S 177 / 5$, n. 3), per i casi più gravi.

Altra limitazione del campo applicativo delle due ipotesi criminose in esame deriva dal fatto che alle condotte di abuso sessuale a danno di soggetti affetti da malattie o handicap (dunque incapaci per ragioni non 'transitorie') si applica l'autonomo delitto aggravato di cui al § $177 / 4$ StGB.

Alla luce di ciò, ne deriva che le previsioni incriminatrici di cui sopra si applicheranno ad ipotesi in cui la capacità di formare o manifestare il dissenso sia assente o fortemente circoscritta per ragioni 'transitorie', come ad esempio uno stato di sonnolenza, una condizione di ubriachezza o di obnubilamento da uso stupefacenti, e fors'anche una situazione di perdita della coscienza per cause diverse dalle malattie o dagli handicap (es.: svenimento). In casi poi di assembramento e calca di persone, si potrebbe altresì prospettare l'applicazione del § 177/1, n. 1) StGB, avendo però cura di distinguerlo dall'ipotesi di atti sessuali repentini di cui al n. 3) (pur in presenza di una equiparazione sanzionatoria). Per quanto riguarda la differenziazione tra le previsioni di cui al n. 1) ed al n. 2) del $\S 177 / 1$, è da rimarcare come nel primo si richieda una capacità totalmente assente di formare o esprimere una volontà contraria (es.: uno stato di sonno, ove il soggetto non si svegli), mentre nel senso una capacità "fortemente limitata", in presenza della quale il 'consenso sessuale' sia apparentemente ottenuto, ma ovviamente non sia legalmente valido (si pensi a persone in stato di ubriachezza).

\subsubsection{Le aggressioni sessuale mediante sorpresa ( $\$ 177 / 2, n$. 3 StGB)}

La - forse fin troppo - ampia elencazione delle ipotesi criminose equiparate agli "atti sessuali dissensuali” del $\S 177 / 2$ StGB continua con una delle maggiori novità della 50 . legge di riforma penale, e cioè l'incriminazione degli "atti sessuali a sorpresa", di cui al n. 3) della norma citata. Tale previsione è senz'altro una di quelle che rivela la maggiore 'connessione' con i fatti del Capodanno 2016 a Colonia ed Amburgo, caratterizzati da numerose aggressioni sessuali 
perpetrate da gruppi di uomini sfruttando la confusione, l'assembramento e, appunto, il fattore sorpresa.

Nelle ipotesi in esame, dunque, l'agente non realizza gli atti sessuali in presenza di un dissenso reale della vittima, e neanche sfruttando una condizione di inferiorità psichica o fisica della stessa ${ }^{73}$, bensì si avvale di una condotta di intrusione sessuale rapida a fronte della quale il soggetto passivo non ha il tempo di esprimere la propria contraria volontà. In conseguenza di ciò, è chiaro come - ove il soggetto sia pienamente capace, e non sia sottoposto a "costrizione ambientale" - dopo tale approccio sessuale iniziale, possibilmente sfociante in un toccamento dei glutei, del seno o delle parti intime della vittima, la situazione si evolverà di regola con le seguenti modalità:

1) La vittima esprime il proprio dissenso, magari respingendo fisicamente l'aggressore e quest'ultimo persiste nel compimento degli atti sessuali: in tal caso quasi certamente integrerebbe uno o più dei delitti delitti aggravati di cui ai §§ 177/5 - 177/8 (es.: atti sessuali violenti, atti sessuali penetrativi ecc.), così che la figura criminosa in esame risulterebbe assorbita.

2) La vittima presta il proprio consenso agli atti sessuali: in tal caso, al di là della questione sulla punibilità dell'approccio iniziale ${ }^{74}$, tendenzialmente la vicenda non verrebbe portata all'attenzione degli organi inquirenti.

3) L'agente, spontaneamente o a seguito del manifestato dissenso del soggetto passivo, interrompe senza indugi l'approccio sessuale. Sono proprio queste le ipotesi concrete cui la nuova incriminazione appare rivolgersi, posta l'assenza di violenza, minaccia o dissenso (il quale sarebbe manifestato dopo, e non prima).

Volgendo lo sguardo a sud delle Alpi, tali casi sono già da quasi due decenni pacificamente qualificati come "atti sessuali violenti" ex art. 609-bis/1 c.p. dalla giurisprudenza italiana di legittimità, pur se quasi sempre collocati tra le ipotesi di minore gravità (pena ridotta fino a 2/3 ai sensi dell'art. 609-bis/3): il BGH tedesco però, come si è già detto, ha sempre interpretato il concetto di "violenza" in modo maggiormente conforme al principio di legalità, facendo prevalere quest'ultimo sulle innegabili esigenze di giustizia sostanziale che spingevano in senso opposto.

\footnotetext{
${ }^{73}$ Seppur in casi concreti di atti sessuali commessi approfittandosi di una situazione di assembramento di persone, possa configurarsi una potenziale applicazione altresì del $\S 177 / 1, \mathrm{n}$. 1), appena commentato.

${ }^{74}$ Al riguardo, soprattutto in contesti di relazione di coppia, vi potrebbero essere ampi margini per configurare una onesta "erronea supposizione del consenso".
} 


\subsubsection{Le aggressioni sessuali 'minacciose' (\$ 177/2, nn. 4 e 5)}

Le ulteriori due fattispecie criminose poste a chiusura - nn. 4) e 5) - del § 177/2 StGB (dunque anch'esse destinatarie di una forbice edittale tra 6 mesi e 5 anni di reclusioni) comportano l'incriminazione di aggressioni sessuali lato sensu connotate da minaccia, la quale però non deve attingere il livello di gravità di cui alle ipotesi del § 177/5.

La previsione incriminatrice di cui al n. 4) sanziona chiunque realizzi atti sessuali "approfittandosi di una situazione nella quale la vittima tema di subire un male rilevante in caso di resistenza". Siffatta previsione è all'apparenza del tutto innovativa, ma in realtà è stretta parente di quella Ausnutzungsvariante introdotta con la legge di riforma del 1997, ed ora collocata quale ipotesi di aggressione sessuale aggravata sotto il $\S 177 / 5$, n. 3) StGB, per la quale occorre "una situazione in cui la vittima sia indifesa alla mercé dell'agente". Senza ripetere quanto già detto nei paragrafi precedenti, va ricordato come tale figura criminosa mirasse a rendere penalmente tipici atti sessuali perpetrati - in assenza di una vera e propria minaccia o violenza - avvalendosi di una situazione di c.d. "costrizione ambientale". La fattispecie introdotta con la riforma del 2016 (§ 177/2, n. 4) si distingue peraltro da quest'ultima in quanto presuppone una "costrizione ambientale" indubbiamente più lieve, non richiedendosi né che la vittima sia indifesa, né che sia alla mercé dell'agente, ma occorrendo unicamente che il soggetto passivo si trovi in una situazione in cui tema di subire un male rilevante in caso di resistenza. Si può dunque pensare ad una sua applicazione in situazioni concrete in cui la persona destinataria dell'approccio sessuale - pur non versando in una condizione psico-fisica di terrore paralizzante (magari accompagnata da un isolamento spaziale e dall'impossibilità di ottenere soccorso tempestivo) - tema fondatamente per la sua incolumità in caso di resistenza, alla luce magari del comportamento attuale e/o passato dell'agente (es.: ex partner già responsabile di condotte di stalking). In definitiva, sarà l'intensità dei 'fattori coartanti' a fungere da discrimine. Ciò tra l'altro corrisponde all'intento legislativo, posto che nella relazione di accompagnamento si parla di "situazione di minorata difesa meramente soggettiva"75, cioè non fondata su indici oggettivi (come invece è richiesto dalla disposizione ex $\S 177 / 5$, n. 3, StGB).

Senz'altro non innovativa è invece l'ultima figura criminosa contemplata dal § 177/2, n. 5) StGB,e cioè quella degli atti sessuali costrittivi commessi "mediante minaccia di un male rilevante". Qui ci si trova di fronte ad una classica ipotesi di costrizione sessuale mediante

75 “Lediglich subjektiv schutzlose Lage", v. Relazione di accompagnamento al progetto di legge governativo 18/8210, cit., p. 11. 
minaccia, la quale si applicherà peraltro solo ove il male prospettato non consista in un “pericolo attuale per la vita o l'integrità fisica" della vittima, ravvisandosi in tale ultima evenienza l'integrazione della fattispecie aggravata di cui al §177/5, n. 2) StGB (con pena da 1 a 15 anni di reclusione).

\subsection{Lo "Stupro" e gli altri delitti sessuali di maggiore gravità di cui ai $\$ \S 177 / 4$ - $177 / 8$ StGB}

I delitti tipizzati dai nuovi - in quanto introdotti dalla riforma del 2016 - $\$ \S 177 / 4$, 177/5, 177/6, 177/7 e 177/8 StGB, tutti caratterizzati da una pena massima di 15 anni di reclusione (a differenza delle ipotesi dei primi 2 Absätze, il cui massimo edittale è 5 anni), ripropongono in una scala di gravità crescente le tipologie di "aggressione sessuale" e "abuso sessuale” già contemplate dai previgenti §§ 177 e 179 StGB. Per quanto riguarda le fattispecie sanzionate con la reclusione da un minimo di 1 o 2 anni fino ad un massimo di 15 anni, vanno ribadite le critiche sull'eccessiva ampiezza di tali forbici edittali.

In considerazione del loro carattere non innovativo, ci si limiterà a delinearne i principali tratti distintivi, seguendo per la trattazione il medesimo ordine del Testo punitivo tedesco, e rimandando ai paragrafi precedenti - e agli autori ivi citati in nota - per ulteriori approfondimenti.

$\$ 177 / 4$ - Atti sessuali con persona affetta da malattia o handicap che le impedisca di formare o esprimere il consenso agli stessi. (Pena prevista: da 1 a 15 anni di reclusione).

La figura criminosa in esame ripropone i contenuti del previgente delitto di “Abuso sessuale di persona incapace di resistere" di cui al $\S 179 / 1$, n. 1) StGB (ora abolito), con una formulazione nella sostanza identica. L'unica differenza rilevante è data dall'inasprimento sanzionatorio rispetto alla forbice (da 6 mesi a 10 anni di reclusione) precedentemente contemplata: la stessa è peraltro dovuta al fatto che le ipotesi meno gravi di abuso sessuale ai danni di soggetti fisicamente o psichicamente incapaci saranno ora sanzionate ai sensi del già commentato nuovo delitto di cui al $\$ 177 / 2$, n. 2), StGB, con pena da 6 mesi a 5 anni.

$\S 177 / 5$ - Atti sessuali commessi mediante violenza, minaccia grave (di un pericolo attuale per la vita o l'integrità fisica della vittima), o approfittamento di una situazione in cui la vittima è indifesa alla mercé dell'agente. (Pena prevista: da 1 a 15 anni di reclusione). 
Tale disposizione ripropone - sanzionandole allo stesso modo - le medesime ipotesi criminose di cui al $1^{\circ}$ Abs. del vecchio $§ 177$ StGB ("Costrizione sessuale / Stupro"), nella versione vigente dal 1997 al 2016. Sarà senz'altro interessante analizzare le prime sentenze del $\mathrm{BGH}$ che si troveranno a dover distinguere in concreto - posta la rilevante discrepanza a livello sanzionatorio - la "minaccia di un pericolo attuale per la vita o l'incolumità fisica" di cui alla presente norma dalla "minaccia di un male rilevante" di cui al § 177/2, n. 5 StGB (il cui massimo edittale è 5 anni di reclusione anziché 15); ma soprattutto a differenziare la "situazione in cui la vittima è indifesa alla mercé dell'agente", di cui al $\$ 177 / 5$, dalla "situazione nella quale la vittima tema di subire un male rilevante in caso di resistenza" di cui al § 177/2, n. 4 StGB (il cui massimo edittale è anch'esso tre volte inferiore).

$\underline{\S 177 / 6 \text { - Casi di particolare gravità (senza pretesa di esaustività vengono }}$ espressamente menzionati - secondo la tecnica degli "esempi di norma" - gli atti sessuali penetrativi e gli atti sessuali commessi da più persone concorrenti). (Pena prevista: da 2 a 15 anni di reclusione).

Anche la suddetta previsione non presenta, sia a livello di tipicità che di scelte sanzionatorie, alcun carattere innovativo rispetto alla disciplina in vigore anteriormente alla riforma. Possono pertanto riproporsi - tra le altre - le medesime osservazioni critiche relative all'incongruità del minimo edittale di soli 2 anni contemplato per le ipotesi di stupro o di aggressione sessuale di gruppo, che nell'ordinamento italiano sono sanzionate rispettivamente con un minimo di 5 (art. 609-bis c.p.) e 6 anni di reclusione (art. 609-octies c.p.): per quanto concerne le aggressioni sessuali di gruppo, tra l'altro, il rischio di sanzionare troppo severamente atti sessuali blandi è ora - a seguito della riforma - senz'altro più ridotto, vista l'introduzione del nuovo reato di “Molestie sessuali” ( $\$ 184 \mathrm{i} \mathrm{StGB}$ ), il quale contempla un' ipotesi aggravata applicabile alle molestie di gruppo (§ 184i/2).

§177/7 - Aggressioni sessuali connotate dal porto di armi finalizzato a impedire la resistenza della vittima; aggressioni sessuali nelle quali la vittima venga posta in grave pericolo di subire rilevanti danni alla salute. (Pena prevista: da 3 a 15 anni di reclusione).

$\S 177 / 8$ - Aggressioni sessuali durante le quali l'agente faccia uso di armi; aggressioni sessuali durante le quali la vittima subisca gravi maltrattamenti o venga a trovarsi in pericolo di vita. (Pena prevista: da 5 a 15 anni di reclusione).

Trattasi di figure criminose aggravate (autonomamente, non prevedendo lo StGB, a differenza del Codice Rocco, il delitto circostanziato) oramai da anni vigenti nell'ordinamento penale tedesco, in relazione alle quali si porrà spesso la necessità di applicare gli istituti relativi 
al concorso di reati e di pene di cui ai §§ 52 - 55 StGB, in base ai quali la pena complessivamente irrogata non potrà comunque oltrepassare gli anni 15 di reclusione. Tale limite potrà invece essere superato ove venga integrato il delitto di cui al § 178 StGB, intitolato "Aggressione sessuale, Costrizione sessuale e Stupro con esito mortale"76: ove l'agente, quanto meno colposamente, cagioni mediante l'aggressione sessuale la morte della vittima, lo stesso sarà punito con la pena dell'ergastolo oppure della reclusione da 10 a 15 anni.

\subsection{Le fattispecie (autonome) attenuate del § $177 / 9$}

In chiusura della norma 'omnibus' di cui al nuovo § 177 StGB è inserita la previsione (§ 177/9) concernente i “casi di minore gravità". Il diritto penale tedesco, invero, come già detto non contempla reati circostanziati, e pertanto - già prima della riforma del 2016 - era emersa la necessità di prevedere autonome fattispecie criminose attenuate applicabili alle aggressioni ed abusi sessuali di minore gravità. Visto l'ampio numero di ipotesi criminose previste dal nuovo § 177 StGB, il legislatore d'oltralpe del 2016 ha deciso di elevare da due a tre i livelli sanzionatori e dunque le autonome previsioni criminose - previsti dal § 177/9 per i "casi di minore gravità":

1) Reclusione da 3 mesi a 3 anni per le aggressioni e abusi sessuali (dissensuali, o comunque non caratterizzati da violenza o minaccia / abuso gravi) di cui ai $\$ \S 177 / 1$ e $177 / 2$ StGB.

2) Reclusione da 6 mesi a 10 anni per le aggressioni e abusi sessuali (connotati da violenza, o minaccia / abuso gravi) di cui ai §§ 177/4 e 177/5 StGB.

3) Reclusione da 1 anno a 10 anni per le aggressioni sessuali (accompagnate dall'uso di armi, inflizione di gravi maltrattamenti ecc.) di cui ai §§ 177/7 e 177/8 StGB.

Da osservare che, logicamente, non è prevista una variante di 'minore gravità' per le ipotesi aggravate di aggressione/abuso sessuale di cui al § 177/6 StGB (atti sessuali penetrativi, di gruppo ecc.).

Ad avviso dello scrivente, tuttavia, alla luce dell'abbassamento a 6 mesi della pena minima per le aggressioni / abusi sessuali meno gravi (§§ 177/1 e 177/2 StGB) operato dal legislatore riformista, non vi era - quanto meno per le figure criminose appena menzionato - una effettiva esigenza di configurare altresì una forbice edittale ulteriormente mitigata per i casi di minore gravità: ciò a maggior ragione se si considera che la stessa 50 . legge di modifica del

76 § 178 StGB: "Sexueller Übergriff, sexuelle Nötigung und Vergewaltigung mit Todesfolge". 
codice penale tedesco ha introdotto - per le intrusioni sessuali più blande - il nuovo delitto di "Molestie sessuali”.

\subsection{Il nuovo delitto di “Molestie sessuali” ex $\$ 184 i$ StGB}

Alle neo-introdotte - e probabilmente 'ipertrofiche' - fattispecie criminose di aggressione ed abuso sessuale di cui al § 177 StGB, l'intervento legislativo di riforma in esame ha affiancato due nuovi delitti di “Molestie sessuali” (§§ 184i/1 e 184i/2 StGB), oltre alla 'anomala' figura criminosa ex § 184j.

Partendo dal reato - mai comparso prima nel Sexualstrafrecht tedesco - di "Molestie sessuali”, il § 184i/1 StGB dispone la pena della reclusione fino a 2 anni (in alternativa alla multa), per chiunque "molesti un'altra persona toccandola fisicamente in maniera sessualmente connotata"77. Il $\$ 184 / i / 2$, poi, contempla una fattispecie aggravata di "Molestie sessuali”, tipizzata adottando la medesima tecnica del "esempio di norma” (“Regelbeispiel”) già utilizzata dal § $177 / 6$ StGB per sanzionare più gravemente gli atti sessuali penetrativi: tale norma prescrive difatti la pena detentiva da 3 mesi a 5 anni nei "casi di particolare gravità", menzionando tra di essi unicamente "la commissione del fatto da parte di una pluralità di persone”. Leggendo la previsione de quo in relazione alla clausola di sussidiarietà di cui al § $184 \mathrm{i} / 1$, in virtù della quale $\mathrm{i}$ reati in esame si applicano solo ove non possano trovare applicazione i più gravi delitti sessuali di cui ai §§ 177/1 ss., è chiaro come difficilmente si potranno ravvisare casi di "molestie sessuali di particolare gravità" diverse da quelle di gruppo.

Focalizzando le attenzioni sulla condotta tipica, la stessa - tipizzata con una formula inevitabilmente un pò indeterminata ${ }^{78}$ - è incentrata su comportamenti molesti consistenti in "toccamenti fisici sessualmente connotati". Ricordandosi quanto detto in sede di trattazione della giurisprudenza tedesca sul concetto di "atti sessuali" (sul quale si imperniano i delitti sessuali 'maggiori', rispetto ai quali il § 184 i espressamente si pone come 'norma residuale'), si può ipotizzare che la qualificazione di "Molestie sessuali" verrà attribuita ad atti sessuali in precedenza qualificati “non rilevanti” ai sensi della norma definitoria di cui al § $184 \mathrm{f} \mathrm{StGB}$, come

\footnotetext{
77 § 184i/1 StGB: "Wer eine andere Person in sexuell bestimmter Weise körperlich berührt und dadurch belästigt".

${ }^{78}$ Come peraltro è tipico di quasi tutti gli ordinamenti che prevedono reati analoghi, vista l'eterogeneità dei comportamenti sessualmente (e non) molesti: cfr, ad esempio l'ipotesi di "Harcèlement sexuel" ex art. 222-33 del codice transalpino, o le norma di cui al britannico "Protection from Harassment Act" 1997.
} 
ad esempio baci superficiali delle labbra, toccamenti dei glutei, palpeggiamenti delle gambe e delle zone vicine al seno, abbracci intensi sgraditi ecc.

In tale contesto, è senz'altro da salutare con favore l'introduzione di una nuova fattispecie tale da racchiudere in sé condotte senz'altro caratterizzate da una spiccata lesività che prima spesso sfuggivano, oltralpe, alla scure del Magistero penale. Ancora più condivisibile, inoltre, è la decisione del legislatore tedesco di tipizzare - pur con la tecnica del Regelbeispiel un'autonoma fattispecie di “Molestie sessuali di gruppo", sanzionata con una pena massima molto più severa dell'ipotesi base ( 5 anni anziché 2 ).

Ciò nonostante, in questa sede non ci si può esimere dall'esprimere alcune osservazioni critiche nei confronti delle scelte normative adottate con la 50. legge di modifica dello StGB, e segnatamente:

1) La norma incriminatrice di cui al $\S 184 i$, fortemente condizionata dall'esigenza di reprimere comportamenti di molestia sessuale perpetrati da sconosciuti (e sovente in gruppo), come quelli avvenuti a Colonia e Amburgo il 1. gennaio 2016, non ha tenuto conto - mediante apposita fattispecie aggravata, e salva la possibile applicazione dei "casi di particolare gravità" di cui al § 184i/2 - del pernicioso e diffuso fenomeno delle molestie sessuali sui luoghi di lavoro.

2) La disciplina in esame ha omesso l'incriminazione delle molestie sessuali non caratterizzate dal coinvolgimento della corporeità sessuale della persona offesa: si pensi ad esempio alla masturbazione molesta dell'agente di fronte alla vittima, spesso concretamente più lesiva, per quest'ultima, rispetto a fugaci toccamenti (es dei glutei).

3) Così come per i delitti sessuali di cui ai §§ $177 / 1$ e 177/2, le pene previste per le molestie sessuali (non di gruppo) dal $§ 184 i / 1$ sono eccessivamente miti (da 3 mesi a 2 anni di reclusione, in alternativa alla multa).

\subsection{La controversa incriminazione della "partecipazione indiretta" ai reati sessuali di gruppo di cui al 184 j StGB.}

La disposizione senz'altro più discutibile è però quella - del tutto peculiare nel panorama giuridico europeo - di cui al nuovo § 184j StGB, dedicato alle molestie (ma anche alle altre aggressioni e abusi sessuali ex $§ 177 \mathrm{StGB}$ ) commesse in gruppo (rectius: dal membro di un gruppo). La suddetta norma prevede infatti un' ipotesi di concorso nel reato (sessuale) che sembra deviare fortemente dai principi in materia di "autoria e partecipazione" sanciti dalla 
parte generale dello Strafgesetzbuch ( $\$ \S 25$ - 31): si contempla invero una pena detentiva fino a 2 anni (o pecuniaria) a carico di chiunque "contribuisca alla commissione di un reato [sessuale] partecipando ad un gruppo di persone il quale induca[letteralmente: spinga/pressioni] un'altra persona alla commissione di un reato [sessuale]", a patto che tali condotte non integrino già di per sé un diverso reato più gravemente punito ${ }^{79}$.

L'unico punto chiaro - emergente dalla disamina delle relazioni di accompagnamento ai progetti di legge già più volte menzionati - è quello concernente le finalità della suddetta norma, consistenti nel garantire una più adeguata repressione delle aggressioni sessuali di gruppo verificantesi in contesti 'concitati' e affollati come quelli del Capodanno di Colonia e Amburgo (situazioni in cui è sovente molto arduo accertare le concrete condotte poste in essere da ciascun membro del 'branco').

Sotto il profilo tecnico, tuttavia, la soluzione normativa adottata lascia quantomeno perplessi: si è difatti scelto di introdurre una sorta di reato di "Partecipazione indiretta ai delitti sessuali di cui ai §§ 177 o 184i StGB". Si può del resto parlare solo di "partecipazione indiretta", posto che quella 'diretta' è già contemplata dalla disciplina sul concorso di persone nel reato di parte generale, in virtù della quale la responsabilità penale - seguendo gli enunciati della teoria della "accessorietà" 80 - richiede necessariamente uno dei tre seguenti legami con il 'fatto-reato' ('Straftat'):

1) Autoria [e coautoria] (Täterschaft, § $25 \mathrm{StGB}$ ).

2) Compartecipazione mediante istigazione (Anstiftung, § 26).

3) Compartecipazione mediante agevolazione (Beihilfe, § 27).

Tenendo presente, inoltre, che l'istigatore è soggetto alla medesima pena dell'autore, mentre per l'agevolatore è prevista unicamente un'attenuazione della stessa (del 25\% per quanto concerne il massimo edittale, ex $\S 49 / 2 \mathrm{StGB}$ ), è del tutto evidente come l'incriminazione di cui al § 184j StGB - applicabile solo ai reati sessuali summenzionati e munita di una cornice edittale affatto differenziata - sanzioni condotte prive delle connessioni con il 'fatto-reato' enunciate dai §§ 25-27 StGB.

\footnotetext{
79 § 184j StGB: "Wer eine Straftat dadurch fördert, dass er sich an einer Personengruppe beteiligt, die eine andere Person zur Begehung einer Straftat an ihr bedrängt wird mit Freiheitsstrafe bis zu zwei Jahren oder mit Geldstrafe bestraft, wenn von einem Beteiligten der Gruppe eine Straftat nach den §§ 177 oder 184i begangen wird und die Tat nicht in anderen Vorschriften mit schwererer Strafe bedroht ist".

80 Secondo tale costruzione dogmatica, la tipicità della condotta principale si estende alle condotte accessorie, in sé atipiche, realizzate dai compartecipi. Non potendosi indugiare oltre sul tema, si rimanda tra i più recenti studi - ex plurimis - a BIANCHI, Manuel. Concorso di persone e reati accessori. Torino: Giappichelli, 2013, p. 128.
} 
Da ciò discende pertanto che il legislatore tedesco abbia inteso, con tale figura criminosa, sancire la rilevanza penale (pur con una pena meno severa) della mera partecipazione ad un gruppo in seno al quale poi uno o più membri vengano indotti alla commissione di uno dei delitti sessuali di cui ai $\S \S 177$ e 184j StGB. Una siffatta disposizione rappresenta ordunque una strumentalizzazione simbolica del diritto penale, atteso che configura una responsabilità penale per un fatto commesso da altri senza richiedere un qualsivoglia legame con la commissione del reato diverso dalla mera partecipazione ad un gruppo: si pongono dunque seri dubbi sulla costituzionalità della norma in relazione al principio di individualità / personalità della responsabilità penale (desumibile ex artt. 100-104 della Grundgesetz [GG] tedesca) ${ }^{81}$, e fors'anche alla libertà di associazione e riunione (art. 8 GG).

\section{CONCLUSIONE}

Volendo dare una valutazione complessiva della radicale riforma del diritto penale sessuale tedesco del 2016, la stessa non può che essere in definitiva che positiva, pur ravvisandosi taluni profili senz'altro censurabili dell'intervento legislativo commentato, al quale vanno comunque attribuite senz'altro i connotati di una legislazione penale "simbolica".

\subsection{Aspetti positivi}

Partendo peraltro - alla luce del giudizio globalmente positivo espresso - dalle innovazioni condivisibili apportate della riforma legislativa de quo, la novità che senz'altro fa 'pendere la bilancia' - ad avviso dello scrivente - verso una valutazione favorevole è l'introduzione del nuovo reato base di "Atti sessuali dissensuali” (§ 177/1 StGB). Inserendo tale incriminazione nel codice penale, difatti, quello tedesco è stato il primo tra i grandi ordinamenti di Civil Law a incentrare - formalmente, oltre che sostanzialmente - il proprio diritto penale sessuale sul consenso (rectius, volontà) del soggetto passivo, anziché sulla violenza, minaccia o altre modalità costrittive (o di abuso).

\footnotetext{
${ }^{81} \mathrm{E}$ ciò anche ove, in sede applicativa si richiedesse una qualche forma di responsabilità colposa in capo all'agente, posto che i reati sessuali sono puniti unicamente a titolo di dolo. Inoltre va rilevato come non si richieda neppure l'istigazione o agevolazione di un diverso reato (si pensi al concorso anomalo ex art. 116 c.p. italiano).
} 
La suddetta opzione legislativa è stata (ed è) sì caratterizzata da un forte impatto simbolico - espresso icasticamente dal diffuso slogan "No significa no", che i media e la società civile teutonici hanno associato alla riforma - , ma al contempo è apprezzabile sotto il profilo funzionale, distinguendosi pertanto da altre norme penali ( presenti - come si vedrà - anche nella medesima legge) nelle quali l'aspetto simbolico non è affiancato da un effettivo e/o ragionevole potenziamento della tutela penale delle vittime. Sancire la rilevanza penale di qualunque atto sessuale realizzato contro la riconoscibile volontà del destinatario, al di là della funzione generalpreventiva positiva mediante orientamento delle norma in questione (da non sopravvalutare), limiterà in futuro - pur restando gli ineliminabili problemi probatori tipici di taluni casi concreti - le assoluzioni di persone responsabili di approcci sessuali non graditi.

In una tale prospettiva, sono senz'altro da apprezzare due ulteriori modifiche legislative connotate dalla medesima finalità, e cioè l'incriminazione espressa degli "atti sessuali repentini/a sorpresa" ( $\$ 177 / 2$, n. 3 StGB) e l'introduzione del nuovo delitto di "Molestie sessuali” (§ 184i). Una valutazione favorevole, infine, può esprimersi pure in relazione all'ampliamento delle ipotesi di "abuso sessuale" penalmente rilevanti, ed alla qualificazione de facto - delle aggressioni sessuali commesse mediante violenza o minaccia grave come fattispecie autonome con pena edittale più elevata rispetto a quelle meramente 'dissensuali'.

\subsection{Aspetti negativi}

Passando invece ai profili che in questa sede si ritengono criticabili, l'innovazione maggiormente problematica è indubbiamente data dal nuovo reato già etichettato come “Partecipazione indiretta ai reati sessuali" di cui al § 184j StGB.

Con siffatta previsione incriminatrice il legislatore d'oltralpe ha invero optato all'opposto di quanto fatto sancendo la punibilità degli atti sessuali 'dissensuali' - per un modello negativo di 'diritto penale simbolico', nel quale il pur comprensibile intento di conseguire una maggiore tutela delle vittime di molestie e aggressioni sessuali di gruppo si è tradotto in una disposizione chiaramente in contrasto con $i$ principi fondamentali - dotati di rilevanza costituzionale anche in Germania - del diritto penale, configurando una sorta di responsabilità penale 'di posizione' data dall'appartenenza ad un gruppo di cui uno dei membri commetta uno dei reati sessuali di cui ai §§ 177 o 184i StGB. 


\section{DIREITO}

LA RIFORMA DEI REATI SESSUALI IN FERMANIA DEL 2016

Altri profili che suscitano perplessità - seppure in misura inferiore - sono invece $i$ seguenti:

1) L'eccessiva proliferazione di nuove fattispecie di abuso e aggressione sessuale, soprattutto in riferimento alle ben 5 distinte figure criminose - equiparate agli "atti sessuali dissensuali" - di cui al § 177/2 StGB.

2) La mitezza dei limiti edittali minimi (6 mesi di reclusione nelle ipotesi 'base', 1 anno per gli atti sessuali commessi mediante violenza o minaccia grave), unita ad una eccessiva dilatazione di forbici edittali (già presente ante reforma, ma ora acuita) che - salvo i §§ 177/1 e $177 / 2$ StGB - si estendono fino a 15 anni di reclusione.

3) La previsione, al § 177/9 StGB di ipotesi autonomamente attenuate ("casi di minore gravità") di reati sessuali già sanzionati con una pena minima di 6 mesi di reclusione, che porranno senz'altro criticità applicative ai fini della distinzione rispetto alla neo-introdotta incriminazione di "Molestie sessuali” (§ 184i StGB).

4) La non incriminazione delle molestie sessuali non fisiche e la mancata autonoma considerazione (con pena aggravata, e salvo applicazione del $\S 184 i / 2$ StGB, quale "caso di maggiore gravità") delle molestie sessuali sui luoghi di lavoro.

\section{BIBLIOGRAFIA}

BIANCHI, Manuel. Concorso di persone e reati accessori. Torino: Giappichelli, 2013.

CARD Richard; GILLESPIE Alisdair; HIRST, Michael. Sexual Offences. Bristol: Jordan, 2008.

EISELE, Jörg. Die Regelbeispielsmethode im Strafrecht. Tuebingen: Mohr Siebeck, 2004.

FASSIN, Bernhard. Strazumessung im Urteilstenor: Regelbeispiele besonders und minder schwerer Fälle. Shaker Verlag: Aachen, 1995.

FISCHER, Thomas. Zum letzen Mal: Nein heißt Nein. Zeit online, 28 giugno 2016. Disponibile in http://www.zeit.de/gesellschaft/zeitgeschehen/2016-06/rechtspolitik-sexualstrafrecht-neinheisst-nein-fischer-im-recht. Acesso em: 10 out. 2017.

FOLKERS, Susanne. Ausgewaehlte Probleme bei sexueller Noetigung und Vergewaltigung aus der Sicht der Praxis. Baden Baden: Nomos, 2004.

FROMMEL, Monika. Das neue Gesetz ist “Unsinn”. Deutschlandradio Kultur: 7 luglio 2016. Disponibile in: http: / /www.deutschlandradiokultur.de/sexualstrafrecht-das-neue-gesetz-istunsinn.1008.de.html?dram:article_id=359391. Acesso: 10 out. 2017. 
FROMMEL, Monika. Die Reform der Reform der Reform ...des Sexualstrafrechts - eine endlose Geschichte. NK, 2003.

FROMMEL, Monika. Das klägliche Ende der Reform der sexuellen Gewaltdelikte. ZRP, 1988.

GÖSSEL, Karl Heinz. Das neue Sexualsfrafrecht. Berlin: De Gruyter, 2005.

HARBECK, Birgit. Probleme des Einheitstatbestandes sexueller Nötigung/ Vergewaltigung. Baden Baden: Nomos, 2001.

HELMKEN, Dierk. Vergewaltigungsreform und kein Ende?. ZRP, 1995.

HÖRNLE, Tatjana. Kopfschütteln oder Weinen genügt. Taz.de: 5 luglio 2016. Disponibile in http://www.taz.de/!5315782/. Acesso em 10 out. 2017.

HOVEN, Elisa; FISCHER, Thomas. Wir erleben eine Moralisierung des Rechts. LTO - Legal Tribune Online, 31 ottobre 2016. Disponibile in http: //www.lto.de/recht/hintergruende/h/sexualstrafrecht-reform-elisa-hoven-thmoasfischer/. Acesso em 10 out. 2017.

KIELER, Marita. Tatbestandsprobleme der sexuellen Nötigung, Vergewaltigung sowie des sexuellen Missbrauchs widerstandsunfähiger Personen, Berlin: Tenea, 2003.

LAUBENTHAL, Klaus. Sexualstraftaten, Heidelberg: Springen, 2000.

LENCKNER, Theodor. § $184 \mathrm{f}$ StGB. In: SCHÖNKE, Adolf; SCHRÖDER, Hoerst; LENCKNER Theodor. Strafgesetzbuch Kommentar, 27a Ed. München: Beck, 2006.

MACRÌ, Francesco. La violenza sessuale (art. 609-bis c.p.) nella giurisprudenza della Suprema Corte del 2015. Diritto penale contemporaneo (rivista trimestrale), $n^{\circ}$ 1, 2016.

MACRÌ, Francesco. Verso un nuovo diritto penale sessuale. Firenze: FUP, 2010.

MACRì, Francesco. Costrizione 'ambientale' agli atti sessuali: la tutela del dissenso tra legalità ed esigenze repressive in un raffronto tra codice penale italiano e StGB tedesco. Rivista Italiana Diritto e Procedura Penale, $n^{\circ}$ 4, 2007.

MILDERBERGER, Elke. Schutzlos - Hilflos - Widerstandsunfähig: Einige Anmerkungen zur Auslegung der Tatbestandserweiterung des \$ 177 StGB n. F. Muenster: LIT.

MÜLLER, Henning Ernst. Der "erkennbare” Wille nach dem neuen Sexualstrafrechts erkennbar fehlerhaft. Pubblicato l'8 luglio 2016 e disponibile in www.community.beck.de. Acesso em 10 out.2017.

OPPENHOFF, Friedrich. Das Strafgesetzbuch für das Deutsche Reich, 12a Ed. Berlin: De Gruyter, 1891.

PAPA, Michele; PALAZZO, Francesco. Lezioni di diritto penale comparato. 3a Ed. Torino: Giappichelli, 2013. 
PAVARANI, Cristina. II mero dissenso della vittima nella violenza sessuale: profili di diritto italiano e anglosassone. Ind. pen., 2002.

RENZIKOWSKI, Joachim. Das Sexualstrafrecht nach dem 6. Strafrechtsreformgesetz. NStZ, 1999.

SCHROEDER, Friedrich-Christian. Die Revolution des Sexualstrafrechts 1992 - 1998. JZ, 1999.

SICK, Brigitte. Sexuelles Selbstbestimmungsrecht und Vergewaltigungsbegriff. Berlin: Duncker \& Humblot, 1993.

STRATENWERTH, Guenter; SCHULTZ, Hans. Leitprinzipien der Strafrechtsreform. Köln: Springer, 1970.

WOLTER, Juergen; HORN, Eckhard. § 184f StGB. In: RUDOLPHI, Hans Joachim; HORN, Eckhard; SAMSON, Erich. SK - StGB. 68a Ed. Muenchen: Beck, 2007.

Artigo convidado

\section{COMO FAZER A REFERÊNCIA DO ARTIGO (ABNT):}

MACRI, Francesco. La riforma dei reati sessuali in fermania del 2016. Revista Eletrônica do Curso de Direito da UFSM, Santa Maria, RS, v. 13, n. 1, p. 370-410, abr. 2018. ISSN 1981-3694. Disponível em: <

https: //periodicos.ufsm.br/revistadireito/article/view/32281 >. Acesso em: dia mês. ano. doi: http://dx.doi.org/10.5902/1981369432281 . 